Mens

revue d'histoire intellectuelle de l'Amérique française

\title{
La longue marche de l'Afrique du Sud : en mémoire des Canadiens français qui ont participé à la première intervention militaire du Canada au $\mathrm{XX}^{\mathrm{e}}$ siècle
}

\author{
John MacFarlane
}

Volume 7, numéro 2, printemps 2007

URI : https://id.erudit.org/iderudit/1024124ar

DOI : https://doi.org/10.7202/1024124ar

Aller au sommaire du numéro

Éditeur(s)

Centre de recherche en civilisation canadienne-française

ISSN

1492-8647 (imprimé)

1927-9299 (numérique)

Découvrir la revue

Citer cet article

MacFarlane, J. (2007). La longue marche de l'Afrique du Sud : en mémoire des Canadiens français qui ont participé à la première intervention militaire du Canada au XX ${ }^{\mathrm{e}}$ siècle. Mens, 7(2), 197-240. https://doi.org/10.7202/1024124ar
Résumé de l'article

Dans cet article, nous nous penchons sur la participation des Canadiens français à la Guerre des Boers (1899-1902), ainsi que sur la place de ce conflit dans la mémoire collective. Contrairement à une opinion reçue, il ressort de l'analyse de quotidiens importants et de discours de notables que la politique suivie par le gouvernement de sir Wilfrid Laurier était appuyée avec modération par une majorité de Canadiens français, du moins dans les premiers mois de la guerre, et que plusieurs d'entre eux ont servi sous les drapeaux, parfois avec brio. L'oubli de ces faits dans la mémoire collective est attribuable au préjugé de l'analyse des impérialistes durant le conflit, à l'absence de contribution significative des Canadiens français à la mise sur pied de l'armée régulière du Canada, ainsi qu'au discours nationaliste canadien-français, puis québécois, au $\mathrm{XX}^{\mathrm{e}}$ siècle qui, en mettant l'accent sur les conflits entre francophones et anglophones, tendit à en exagérer la portée. 


\title{
LA LONGUE MARCHE DE L'AFRIQUE DU SUD : EN MÉMOIRE DES CANADIENS FRANÇAIS QUI ONT PARTICIPÉ À LA PREMIÉRE INTERVENTION MILITAIRE DU CANADA AU XX SIÈCLE $^{1}$
}

\author{
John MacFarlane \\ Ministère de la Défense nationale
}

\section{Résumé}

Dans cet article, nous nous penchons sur la participation des Canadiens français à la Guerre des Boers (1899-1902), ainsi que sur la place de ce conflit dans la mémoire collective. Contrairement à une opinion reçue, il ressort de l'analyse de quotidiens importants et de discours de notables que la politique suivie par le gouvernement de sir Wilfrid Laurier était appuyée avec modération par une majorité de Canadiens français, du moins dans les premiers mois de la guerre, et que plusieurs d'entre eux ont servi sous les drapeaux, parfois avec brio. L'oubli de ces faits dans la mémoire collective est attribuable au préjugé de l'analyse des impérialistes durant le conflit, à l'absence de contribution significative des Canadiens français à la mise sur pied de l'armée régulière du Canada, ainsi qu'au discours nationaliste canadien-français, puis québécois, au $\mathrm{XX}^{\mathrm{e}}$ siècle qui, en mettant l'accent sur les conflits entre francophones et anglophones, tendit à en exagérer la portée. 


\section{Summary}

This article examines the French Canadian participation in the Boer War (1899. 1902) and the place that the war occupies in Quebec's collective memory. An analysis of the province's major dailies reveals that, contrary to popular belief, a majority of French Canadians cautiously supported Sir Wilfrid Laurier's war policy, at least during the conflict's first months. Moreover, a number of Frenchspeaking Catholics served in the Canadian contingent, at times with great distinction. Over time, French Canadian support and participation in the Boer War has been obscured by three factors: the enduring legacy of wartime imperialist rhetoric, the relative absence of French Canadian participation in the establishment of Canada's regular forces, and French Canadian (and later Quebec) nationalist discourse, which over-emphasized conflict between Anglophones and Francophones.

I didn't go to the moon, I went much further for time is the longest distance between two places -Tennessee Williams, The Glass Menagerie

Les interventions militaires du $\mathrm{XX}^{\mathrm{e}}$ siècle ne sont généralement pas considérées comme des moments qui ont uni les Canadiens, tout particulièrement au Québec. Ils n'ont pas semblé contribuer à la consolidation de l'identité canadiennefrançaise, puis québécoise, au fil du $\mathrm{XX}^{\mathrm{e}}$ siècle. Il est révélateur que, dans un dossier paru dans le magazine L'Actualité à la fin du siècle précédent sur les 100 Québécois «qui ont fait le $\mathrm{XX}^{\mathrm{e}}$ siècle », les personnalités et événements militaires étaient absents ${ }^{2}$. Étant donné que l'appui à l'armée n'a jamais été unanime et que certains groupes de la population canadienne-française s'y sont toujours vigoureusement opposés, on a eu tendance à reléguer la chose militaire aux oubliettes.

Jacques Mathieu et Jacques Lacoursière font remarquer que le rappel des événements du passé n'est jamais neutre. Ils sont mis au service du présent, pour l'avenir. Si un groupe est 
divisé sur un événement donné, celui-ci ne peut véritablement servir à la consolidation de son identité. Par ailleurs, les deux guerres mondiales ont causé beaucoup de douleur et de souffrance. C'est une raison de plus, dans leur cas, pour refouler leur souvenir ${ }^{3}$. Pourtant, l'oubli des événements militaires ne signifie pas qu'on ne les a pas appuyés à l'époque. Récemment, en juillet 2007, les journalistes évoquaient l'opposition québécoise à la participation canadienne à la campagne d'Afghanistan, oubliant que, en 2001, l'appui à cette mission s'élevait à 78,4 \% ${ }^{4}$. Certains se rappellent peut-être l'appui des Québécois à l'intervention militaire du Canada aux côtés des forces de l'Organisation des nations unies contre l'Irak à la suite de l'invasion du Koweit par l'armée irakienne en $1990^{5}$. Vingt ans plus tôt, en octobre 1970, tout indique qu'ils appuyaient majoritairement l'application de la Loi sur les mesures de guerre, mais peu d'études nous le rappellent, ce qui laisse penser que l'opposition était plus vigoureuse qu'elle l'était en réalitét. En 1950, des journalistes, des hommes politiques, ainsi qu'une bonne partie de la population canadienne-française étaient plutôt favorables à l'intervention militaire en Corée dans le cadre de cette " guerre oubliée ${ }^{7}$ ». Lorsque des historiens du Québec parlent des deux guerres mondiales, ils s'intéressent souvent plus à l'opposition à la conscription qu'à la réaction à la guerre proprement dite, donnant au lecteur l'impression que l'opposition des Canadiens français était vive, alors que, dans chaque cas, l'appui initial à la guerre était majoritaire ${ }^{8}$. Même l'intervention militaire lors des émeutes de 1918 contre la conscription a été fortement appuyée par la population du Québec?. La Guerre des Boers, dans la lointaine Afrique du Sud, est un autre de ces conflits dont, au Québec, on se souvient surtout pour l'opposition qu'il a suscitée. 
Certes, les Canadiens, comme les Britanniques, n'étaient pas tous acquis à la cause impériale en Afrique du Sud, et les Canadiens français comptaient naturellement parmi les plus réticents devant cette entreprise. Mais leur condamnation était-elle aussi unanime qu'on le prétend généralement ? C'est à cette question que nous tâchons de répondre dans cet article. Nous nous penchons dans un premier temps sur l'envoi des contingents canadiens, puis sur la réaction des Canadiens français devant la guerre. Comme pour les autres interventions militaires du XXe siècle, les articles de presse, les débats parlementaires, les déclarations publiques et la correspondance privée de personnalités témoignent d'une acceptation initiale, voire d'un soutien manifeste à l'égard de la décision du premier ministre Wilfrid Laurier d'envoyer des volontaires canadiens en Afrique du Sud. Dans la troisième partie, nous parlons des combattants canadiens-français, puis nous concluons en voyant comment la guerre a transformé le mouvement impérialiste au Canada, la structure de l'armée régulière et les relations entre francophones et anglophones au pays. Pour comprendre cette transformation, il est tout aussi important de tenir compte de l'appui initial des Canadiens français à la guerre que de leur opposition ultérieure à celle-ci, dont on se souvient plus généralement de nos jours.

\section{L'envoi de troupes}

La décision du gouvernement du Canada de participer à la guerre de la Grande-Bretagne contre les Boers d'Afrique du Sud en octobre 1899 n'a pas été prise à la légère. Ottawa hésitait, comme on peut le comprendre, à approuver la politique de Londres. L'armée britannique était mobilisée dans le but manifeste de défendre les sujets de Sa Majesté, ceux que l'on appelait les Uitlanders et qui vivaient dans les colonies boers, mais auxquels les autorités politiques refusaient le droit 
de vote. Le 12 octobre, les Boers, colons d'origine néerlandaise qui avaient coexisté bon gré mal gré avec les Britanniques en Afrique australe tout au long du XIX ${ }^{\mathrm{e}}$ siècle, envahissent le Cap de Bonne-Espérance, britannique, et le Natal, fournissant le prétexte à Londres pour justifier la guerre. Joseph Chamberlain, secrétaire aux Colonies de la Grande-Bretagne, et lord Salisbury, premier ministre anglais, sont impatients de saisir l'occasion de promouvoir le sentiment de solidarité dans l'Empire, et les fidèles de la cause impériale se montrent très désireux de se joindre à la croisade. Certains Canadiens d'origine anglaise dirigent la campagne en faveur de l'envoi de troupes coloniales pour aider la mère patrie. Cependant, beaucoup de ceux qui sont moins sollicités soupçonnent que la liberté que prétend défendre Londres n'est que la liberté de l'homme blanc et anglo-protestant d'exploiter les riches gisements d'or et de diamants de l'Afrique du Sud.

Les motifs pour lesquels les Britanniques se battent en Afrique du Sud sont, en effet, suspects. La découverte de gisements de diamants, en 1867, et d'or, en 1884, suscite énormément d'intérêt pour le Transvaal. En 1898, le haut commissaire britannique, Alfred Milner, se rend en Afrique du Sud, où il cherche à favoriser les intérêts financiers britanniques qui exploitent les gisements de diamants et d'or dans la région. Après la guerre, il prétendra avoir provoqué la crise, inévitable, avant qu'il ne soit trop tard ${ }^{10}$. Milner, comme Chamberlain, espère faire la preuve de la solidarité de l'Empire. Toutefois, ses appels sollicitent davantage les sujets d'un empire intolérant et avide de domination que ceux d'un empire épris de liberté. Par exemple, si les Australiens, les NéoZélandais et les Canadiens sont invités à participer à l'effort de guerre, les troupes offertes par les États fédérés de Malaisie et par le Nigeria sont refusées ${ }^{11}$. Milner, qui compare les 
Canadiens français aux Boers du Cap, ne pensait probablement pas qu'ils participeraient et ne le voulait peut-être pas non plus ${ }^{12}$. Le monde non britannique ne manifeste guère de sympathie pour la cause. Même en Grande-Bretagne, « a strong pro-Boer movement led to trenchant questioning of the aims and methods of both war and imperialism ${ }^{13} \%$.

Parmi les opposants à la guerre, on compte beaucoup de Canadiens d'origine française. Sir Wilfrid Laurier, premier Canadien français à accéder au poste de premier ministre du Canada, sait parfaitement qu'il faut les convaincre qu'Ottawa ne se contentera pas d'entériner une décision prise à Londres. Il s'interroge depuis des semaines, évaluant l'opinion publique et manifestant au moins autant d'opposition que d'appui à l'idée d'une participation du Canada. Pourtant, lorsque la guerre est déclarée, Ottawa annonce presque immédiatement son appui à la décision de Londres et envoie 1000 volontaires. Plus de 6000 autres suivront au cours de cette guerre de deux ans et demi. Pour affaiblir les opposants, Laurier rappelle que les Britanniques, comme ils le préfèrent d'ailleurs, paieront pour les soldats, que seuls des volontaires iront en Afrique du Sud, que les contingents ne créeront pas de précédent pour la participation du Canada à d'autres guerres britanniques et que, par-dessus tout, la cause qu'Ottawa défend est celle des droits civils et de la liberté religieuse.

En septembre 1899, Laurier déclare à son ministre de la Milice et de la Défense, Frederick William Borden, qu'il est opposé à l'envoi de troupes canadiennes et, le 4 octobre, il annonce publiquement que des troupes ne seront pas envoyées sans l'approbation du Parlement. Cependant, il sait parfaitement qu'il doit convaincre le Canada anglais de son attachement, à n'en pas douter sincère, aux institutions et à la politique britanniques ${ }^{14}$. Il sait tout aussi bien qu'il ne convaincra pas les Canadiens français d'accepter les guerres de l'Empire 
en invoquant la solidarité impériale pour la mère patrie ${ }^{15}$. Lorsque Laurier accepte, finalement, d'envoyer un contingent canadien, il invoque la justesse de la cause sur le plan des droits humains fondamentaux : il s'agit de préserver les droits civils et la liberté religieuse des sujets britanniques. Dans sa réponse à la critique d'un prêtre, il précise que les Boers maltraitent non seulement les Uitlanders, mais aussi les noirs et les catholiques $^{16}$. Mais il mentionne rarement le mauvais traitement de catholiques aux auditoires anglais et il passe sous silence sa préoccupation pour le traitement lamentable réservé à la majorité noire en Afrique du Sud lorsqu'il dit au Parlement, en 1901, qu'il y a deux ethnies dans ce pays, les Anglais et les Néerlandais ${ }^{17}$. Laurier change d'avis en raison des pressions exercées par les Canadiens anglais, mais aussi, et c'est tout aussi important, en raison de sa conviction que les Canadiens français toléreront l'envoi de troupes canadiennes en Afrique du Sud.

Après le départ des troupes à la fin d'octobre, les Canadiens anglais continuent d'exercer des pressions pour obtenir l'envoi d'un plus grand nombre de soldats. Cédant à ces pressions, le cabinet accepte d'envoyer un deuxième contingent au début de novembre, une fois de plus sans consulter le Parlement. Les Britanniques n'acceptent pas l'offre tout de suite. Ce n'est qu'en janvier 1900, peu après la «semaine noire», à la mi-décembre, au cours de laquelle les forces britanniques ont perdu trois batailles importantes, que ce deuxième contingent canadien, comptant 1289 soldats, est envoyé. En 1900, l'opinion évolue au sujet de la guerre, notamment après le refus des Boers de Pretoria de se rendre lors de l'entrée des forces britanniques dans la ville en juin. L'épreuve de force rapide et sans bavure qu'on avait prévue se transforme alors en une guérilla longue et difficile. Les demandes de troupes se multiplient et Laurier, toujours hésitant et de plus en plus 
inquiet, continue d'accepter. Le lord Strathcona's Horse (500 cavaliers) part en mars 1900, suivi de la Force constabulaire d'Afrique du Sud (1 248 hommes) en mars 1901, du deuxième bataillon canadien de fusiliers à cheval (901 hommes) en janvier 1902 et de quatre autres. bataillons canadiens de fusiliers à cheval (2036 hommes) juste avant la fin de la guerre, en mai $1902^{18}$. Laurier, qui suit attentivement l'évolution de l'opinion concernant les politiques de l'Empire, est de plus en plus mal à l'aise devant les demandes de troupes supplémentaires $^{19}$. Il dit à lord Minto, le gouverneur général du Canada, que ces demandes répétées ne sont pas judicieuses, parce qu'elles donnent l'impression que l'armée britannique est incompétente $^{20}$.

Certains Canadiens se battent aussi aux côtés des Boers pendant la guerre. Au moins un Canadien anglais de Toronto est fait prisonnier et La Presse, qui estime que les Boers peuvent compter sur 4000 Américains, 6000 Allemands, 2000 Danois et Belges, 1000 Irlandais, 600 Scandinaves et 200 Français, Suisses et Italiens, met en garde contre les dangers de rejoindre les rangs des Boers. Un Franco-Américain optimiste, Victor Gagné, est sûr que les Boers gagneront, parce que, calcule-t-il en décembre 1900, les Français et les Allemands se joignent à la cause par centaines chaque jour ${ }^{21}$.

\section{L'opinion des Canadiens français}

Il n'est pas facile d'évaluer l'opinion publique au cours de cette période où on ne fait pas encore de sondages. Toute tentative en ce sens doit tenir compte du fait que la marge d'erreur risque d'être assez importante. Tout atteste cependant un appui surprenant à la guerre dans bien des milieux. On n'ignore pas que certains anglophones auraient désiré un engagement plus vigoureux et ont accusé les francophones de ne pas en faire assez, mais d'autres anglophones soulignent à 
Laurier que les critiques les plus acerbes accusant le gouvernement de se plier au désir des Canadiens français de limiter l'envoi de troupes ne sont pas représentatives de la majorité : "do not allow the talk of some fire-eaters to deceive you in this matter", lui écrit l'un d'entre eux ${ }^{22}$. Lorsque George Denison, président de la British Empire League au Canada, informe le premier ministre que les anglophones s'agitent, Laurier lui répond : « that may be; but there are other quarters where the public are a good deal restless, not at the delays, but at our actions [...] There is in my province a growing resistance to imperialism, which has been caused by too zealous friends. This fact may be unshown in Ontario, but it is brought every day to my eyes ${ }^{23} »$.

La guerre est l'occasion de faire la preuve de sa fidélité à l'Empire, et Laurier a bien des raisons de refuser que le Canada y participe. Les extrémistes canadiens-anglais expriment bruyamment leur point de vue, mais celui-ci n'est guère répandu. Le Mail and Empire de Toronto, qui est l'une des voix les plus radicales, le reconnaît lorsqu'il affirme, au sujet de l'opinion publique canadienne devant la guerre, que " the public had not shown enough energy. The press of the country had done noble duty, but the masses of the people had been too passive ${ }^{24} »$. La presse canadienne-anglaise, ainsi que la population, est en majorité beaucoup plus posée, attendant la décision de Laurier. En fait, lorsque le premier ministre décide d'envoyer un contingent, c'est une opposition désorganisée qui proteste au Canada anglais ${ }^{25}$. La plupart soutiennent la participation du Canada comme démonstration concrète de patriotisme impérial et de solidarité collective dans tout l'Empire ${ }^{26}$. Comme on pouvait s'y attendre, les extrémistes veulent que Laurier aille plus loin et envoie plus de troupes ou accepte de payer pour des contingents en Afrique du Sud ${ }^{27}$. Loin de s'interroger sur les motifs des Britanniques, ils con- 
centrent leurs attaques sur les Canadiens français et sur le ministre des Travaux publics, Israël Tarte, auxquels ils reprochent leur tiédeur.

Tarte reste opposé à l'envoi de troupes jusqu'à ce que la guerre éclate, puis, le 12 octobre, il finit par accepter la position de son chef, estimant que les francophones toléreront l'intervention si Laurier annonce qu'il ne s'agit pas d'un précédent pour d'autres guerres impériales. Il tolère plus qu'il ne soutient la politique du gouvernement, mais il est loin d'être renégat. En tant que membre de la British Empire League, il aspire à une sorte de fédération impériale, mais il s'indigne des appels à lever des troupes et à donner de l'argent sans aucun droit de représentation aux conseils impériaux ${ }^{28}$. La démission du député libéral Henri Bourassa est regrettable pour les libéraux, mais celle de Tarte serait désastreuse. D'autres francophones opposés à la guerre et sympathiques à la cause de la population boer, non anglaise, rurale et profondément religieuse, semblent suivre Tarte : ils attendaient la décision de Laurier et l'ont acceptée ${ }^{29}$.

Certains appuient même vigoureusement cette décision, parmi lesquels on retrouve des dirigeants politiques, militaires, religieux, économiques et intellectuels du Québec ${ }^{30}$. L'une des raisons pour lesquelles la participation à la guerre est acceptée nous est donnée par l'auteur Louis Fréchette. Selon lui, les Canadiens français sont enclins à soutenir l'Empire dont ils font partie pour obtenir "la confiance et le respect» de leurs concitoyens anglophones, conditions « de l'harmonie entre les différents éléments de notre population, harmonie indispensable au bonheur des nôtres et au développement de notre prospérité nationale $e^{31}$ ». Simon-Napoléon Parent, le maire de Québec, rappelle la loyauté des francophones dans son discours aux troupes qui quittent le pays en octobre. L'influent sénateur libéral Raoul Dandurand, malgré une opposi- 
tion initiale, se rallie lui aussi au mouvement ${ }^{32}$. Louis-Nazaire Bégin, l'archevêque de Québec, affirme que « tout en conservant de l'affection pour notre ancienne mère-patrie [sic], nous [sommes] heureux de vivre à l'ombre du drapeau britannique ». Le président de l'Assemblée législative de l'Ontario, François-Eugène-Alfred Évanturel, est fier de voir que « des enfants de [s]a race sont aujourd'hui à combattre sous le drapeau anglais dans l'Afrique Australe [sic]", et, selon le maire de Montréal Raymond Préfontaine, "nous sommes fiers de voir le Canada fournir autant de vaillants et courageux soldats pour combattre les combats [sic] de l'empire ${ }^{33} »$. Les possibilités accrues de patronage, attribuables aux dépenses de guerre, contribuent peut-être à atténuer l'opposition de certains $^{34}$.

Cependant, les anglophones ne sont peut-être pas très informés de l'ampleur des déclarations d'appui des francophones, minimisées dans certains journaux ${ }^{35}$. De manière générale, l'opposition canadienne-française dans les derniers mois de 1899 n'est pas acharnée et, comme Laurier le laisse entendre, elle est surtout inspirée par l'attitude de critiques anglophones « trop zélés ${ }^{36}$ ». Un citoyen qui écrit à Laurier dit préférer un rapprochement avec la Grande-Bretagne à une union avec les États-Unis, ajoutant que cette guerre injuste ne vaut pas la menace qu'elle représente pour la Confédération, mais que le fait que Laurier a promis que nul ne sera contraint de participer rend la politique tolérable ${ }^{37}$. Au Parlement, la démission d'Henri Bourassa est le signe d'opposition le plus célèbre. Bourassa n'en est pas pour autant radicalement antibritannique ${ }^{38}$. Le jeune député de Labelle précise qu'il est fier de ses droits et de sa liberté, qu'il est loyal à l'Angleterre et qu'il est prêt à défendre le drapeau britannique dans tout le Canada. Or, proteste-t-il, le Canada n'est pas menacé, sauf par le plan de la fédération impériale de Cham- 
berlain et par le précédent que constitue non seulement le fait d'envoyer des troupes, mais le fait de les envoyer sans l'avis du Parlement ${ }^{39}$. La majorité des dirigeants qui s'expriment au nom des Canadiens français continuent néanmoins d'appuyer la politique du gouvernement.

Une analyse de la presse canadienne-française confirme une acceptation étonnante de la décision d'Ottawa à l'automne 1899. Certains petits journaux expriment une opposition agressive, tandis que d'autres manifestent un appui vigoureux à la politique en vigueur. Au Québec, le rédacteur en chef du Soleil, libéral, fait une campagne active aux assemblées publiques en faveur de la participation ${ }^{40}$. Il n'en reste pas moins que la presse anglophone extrémiste attaque souvent les journaux francophones, qu'elle accuse de prendre parti pour les Boers $^{41}$. Deux des journaux les plus souvent incriminés sont La Presse et le journal de Tarte, La Patrie. Comme l'illustre le tableau A, ces journaux, au cours de l'automne 1899, contiennent moins d'articles probritanniques et plus d'articles favorables à la cause des Boers que les journaux anglophones, comme le Montreal Daily Star, un quotidien sympathique aux idées impérialistes. Un article rappelle, par exemple, que le pape est opposé à la guerre et semble sympathique à la cause des Boers ${ }^{42}$. La Presse et La Patrie critiquent aussi ouvertement la politique britannique, faisant valoir que, en essayant d'élargir l'Empire par la force, Chamberlain compromet son unité $^{43}$. Au total, de septembre à décembre 1899, La Presse et La Patrie publient respectivement 29 et 26 articles favorables aux Boers, tandis que 7 et 5 articles critiquent la participation du Canada à la guerre.

Cela dit, pour chaque article critique de la cause britannique, ces journaux publient autant, sinon plus, d'articles favorables au cours de cette période. La Presse et La Patrie publient respectivement 59 et 22 articles favorables aux Britan- 
Tableau A

Réaction de la presse du Québec face à la Guerre des Boers du $1^{\text {er }}$ septembre au 15 décembre 1899

\begin{tabular}{|c|c|c|c|c|c|c|}
\hline Quotidien & $\begin{array}{l}\text { Nombre total } \\
\text { d'articles concernant } \\
\text { la Guerre des Boers }\end{array}$ & $\begin{array}{l}\text { Articles favorables } \\
\text { à la Grande- } \\
\text { Bretagne }\end{array}$ & $\begin{array}{c}\text { Articles } \\
\text { favorables } \\
\text { aux Boers }\end{array}$ & $\begin{array}{l}\text { Articles } \\
\text { neutres }\end{array}$ & $\begin{array}{l}\text { Articles favorables } \\
\text { à la participation } \\
\text { du Canada* }\end{array}$ & $\begin{array}{l}\text { Articles défavorables } \\
\text { à la participation du } \\
\text { Canada* }\end{array}$ \\
\hline $\begin{array}{c}\text { La Presse } \\
\text { (Tirage : } 63000 \\
\text { exemplaires en } 1899^{* *} \text { ) }\end{array}$ & 215 & 59 & 29 & 127 & 35 & 7 \\
\hline $\begin{array}{c}\text { La Patrie } \\
\text { (Tirage : } 27500 \\
\text { exemplaires en } 1901^{* *} \text { ) }\end{array}$ & 197 & 22 & 26 & 149 & 20 & 5 \\
\hline $\begin{array}{c}\text { The Montreal Daily Star } \\
\text { (Tirage : } 51000 \\
\text { exemplaires en } 1899^{* *} \text { ) }\end{array}$ & 435 & 148 & 0 & 287 & 89 & 0 \\
\hline
\end{tabular}

* Les statistiques concernant l'opinion des quotidiens au sujet de la participation du Canada à la Guerre des Boers sont tirées des mêmes textes que ceux qui ont servi à établir les statistiques concernant l'appui à la Grande-Bretagne et aux Boers. C'est pourquoi le nombre total d'articles est inférieur à celui qu'on obtient en additionnant les chiffres de chaque rangée. Pour déterminer si un article était favorable ou non à la participation canadienne, nous avons tenu compte de l'impression générale laissée au lecteur. Par exemple, un article citant un membre du gouvernement, sans commentaire, serait généralement considéré comme favorable. Les éditoriaux de La Presse critiquaient souvent la Grande-Bretagne.

** Les données concernant le tirage de ces quotidiens sont tirées de André Beaulieu et Jean Hamelin, La presse québécoise : des origines à nos jours, Québec, Presses de l’Université Laval, 1973-1990. 
niques, tandis que 35 et 20 articles sont favorables à la participation du Canada à la guerre. Seuls des impéralistes aveuglés par leur partisanerie considéreraient que cette couverture de la guerre témoigne d'un manque de loyalisme. Comme le donnent à penser les articles parus dans le Montreal Daily Star, lorsque l'appui à la guerre est vigoureux, on n'envisage guère l'autre aspect des choses : on y trouve 148 articles nettement favorables à la cause britannique et 89 articles en faveur de la participation du Canada à la guerre. Ce journal publie plus de deux fois plus d'articles sur le sujet que les journaux francophones. Un journaliste de La Presse fait remarquer que «notre devoir est de fournir au public canadien les renseignements les plus exacts [...] où sont concernés [...] l'honneur de l'empire britannique ${ }^{44}$ ", et non de reproduire les articles de la presse à sensation de Londres, comme les journaux jingos. Rappelons que ces deux journaux francophones, tous deux d'obédience libérale, ne sont pas nécessairement représentatifs de l'opinion canadienne-française dans son ensemble. Néanmoins, le fait demeure : les quotidiens francophones les plus lus au Canada français à l'époque soutiennent, de manière modérée, la position du gouvernement. Par ailleurs, le tableau $\mathrm{A}$ indique que l'appui à la cause augmente dès que Laurier annonce publiquement que le Canada participera à la guerre $^{45}$. En somme, la presse francophone commence par s'aligner sur la position britannique, mais pas avec assez d'enthousiasme pour satisfaire les jingos.

Il n'est pas facile de mesurer avec exactitude le degré d'opposition des Canadiens français à la participation du Canada à la guerre. Il n'y a pas eu d'émeutes dans les rues, hormis une rixe mineure en mars $1900^{46}$. À la Chambre des communes, 10 Canadiens français seulement (sur environ 70) appuient la proposition de Bourassa, qui veut que ce soit le Parlement qui décide des futures interventions militaires ou mo- 
difications des relations avec la Grande-Bretagne $e^{47}$. Pour prendre plus précisément le pouls de l'opinion publique, il vaut mieux se tourner vers les élections fédérales de novembre 1900, où sont mises à l'épreuve les théories des extrémistes anglophones (selon qui $99 \%$ des Canadiens appuient la guerre) et des extrémistes francophones (selon qui $99 \%$ des Canadiens français sont tout à fait opposés à la guerre $)^{48}$. Certes, un vote pour Laurier n'est pas nécessairement un vote en faveur de la participation canadienne à la guerre ${ }^{49}$. Néanmoins, les résultats électoraux indiquent bien qu'une majorité de Canadiens préfèrent la politique libérale à celle que défendent les conservateurs et d'autres partis émergents. Si le Parti libéral perd du terrain en Ontario pendant et après la guerre ${ }^{50}$, en revanche, en 1900 (et encore plus en 1904), il obtient un appui croissant au Québec, passant de 49 à 57 sièges (sur 65), ainsi que partout ailleurs au Canada anglais, comme le montre le tableau B. Le chef conservateur, sir Charles Tupper, est même défait dans sa propre circonscription du Cap Breton aux élections de 1900.

Les Canadiens français sont aussi touchés que les autres par l'opposition croissante à la politique britannique à mesure que la guerre se prolonge. Leur loyauté à l'Empire ne peut s'appuyer que sur des critères plus universels que ceux de l'ethnicité et de la culture, comme par exemple la liberté et les droits civils. Or, la politique de la terre brûlée et les camps de concentration mis sur pied par les Britanniques au cours du conflit vont à l'encontre de ces valeurs. Le juge en chef du Cap de Bonne-Espérance, sir John Henry de Villiers, demande à Laurier de l'aider à mettre fin à la guerre injuste que Chamberlain mène pour des raisons économiques. Selon lui, cette guerre ébranle de plus en plus la loyauté des sujets non angloprotestants de l'Empire. Il propose à Laurier de faire entendre à Londres la voix des véritables impérialistes, "who are 
Tableau B

Résultat des élections fédérales de 1896, de 1900 et de 1904 par province et territoire

\begin{tabular}{|c|c|c|c|c|c|c|c|c|c|c|}
\hline Élection & Parti & $\begin{array}{l}\text { Colombie- } \\
\text { Britannique }\end{array}$ & $\begin{array}{l}\text { Île-du-Prince- } \\
\text { Edouard }\end{array}$ & Manitoba & $\begin{array}{l}\text { Nouveau- } \\
\text { Brunswick }\end{array}$ & $\begin{array}{l}\text { Nouvelle- } \\
\text { Écosse }\end{array}$ & Ontario & Québec & $\begin{array}{c}\text { Territoires du } \\
\text { Nord-Ouest }\end{array}$ & TOTAL \\
\hline \multirow{2}{*}{1896} & Libéral & 4 & 2 & 2 & 5 & 10 & 43 & 49 & 3 & 118 \\
\hline & Conservateur & 2 & 3 & 4 & 9 & 10 & 43 & 16 & 1 & 88 \\
\hline \multirow{2}{*}{1900} & Libéral & 4 & 3 & 4 & 9 & 15 & 37 & 57 & 4 & 133 \\
\hline & Conservateur & 2 & 2 & 3 & 5 & 5 & 55 & 8 & 0 & 80 \\
\hline \multirow{2}{*}{1904} & Libéral & 7 & 1 & 7 & 7 & 18 & 38 & 53 & 7 & 138 \\
\hline & Conservateur & 0 & 3 & 3 & 6 & 0 & 48 & 11 & 4 & 75 \\
\hline
\end{tabular}


not inflated by the pride of race or animated by a lust for domination ${ }^{51}$ ». À l'étranger comme au Canada, le point de vue de Laurier sur l'Empire est respecté. Les Canadiens ont eu beaucoup de raisons de s'opposer à la politique britannique en Afrique du Sud. S'ils ont décidé de considérer cette guerre comme une croisade pour la justice et la liberté dans un Empire démocratique, c'est bien grâce à Laurier, le leader canadien-français qui s'est fait le champion de cette interprétation.

Il est révélateur que Bourassa ait parlé en privé d'un sentiment net, mais instable (unsettled), contre l'impérialisme au Québec ${ }^{52}$. Si instable que soit ce sentiment, les francophones paraissent continuer, en majorité, de faire confiance au jugement du chef libéral. Bourassa ne peut pas comprendre cette patience, soutenant que Laurier est plus impérialiste que Chamberlain et que l'impression que Tupper serait pire est une erreur attribuable à la méconnaissance, par les Canadiensfrançais du Québec, du véritable Laurier : « our people naively believe that he is their champion; while, in fact, he is really the greatest danger not only to his compatriots but to the whole of Canada - on account of his weakness and of his perpetual nightmare to be denounced as a traitor ${ }^{53} »$. Le deuxième aspect de l'opinion de Bourassa sur son ancien chef est bien connu et souvent repris par les historiens. Cependant, le premier, c'est-à-dire sa conviction que les Canadiens français appuient la vision de Laurier plutôt que la sienne, est généralement oublié. L'interprétation que Bourassa donne de l'appui des Canadiens français à Laurier aux élections de 1900 semble exacte : "The action of the government has not been approved but excused ${ }^{54}$.» Le soutien des francophones, qui est motivé par des raisons différentes et n'est pas inconditionnel, contraste certes avec l'appui plus net manifesté par 
certaines parties de la population anglophone, mais il n'en est pas moins bien réel.

\section{Les Canadiens français en Afrique du Sud}

On l'oublie souvent : certains francophones ont combattu pour l'Empire dans le cadre de la première expédition du Canada à l'étranger ${ }^{55}$. La légende qui, dans les années 1990, se trouvait à l'entrée de l'exposition du Musée canadien de la guerre commémorant la participation canadienne à ce conflit disait que «lorsque la Grande-Bretagne déclara la guerre aux Boers en octobre 1899, les Canadiens anglais réagirent en levant une armée de volontaires ». Il est vrai que les Canadiens français ne représentent pas une forte proportion du corps expéditionnaire canadien en Afrique du Sud, soit environ $4 \%$, par rapport à leur poids dans l'ensemble canadien, soit environ $30 \%$ à l'époque. Il faut dire qu'outre le fait qu'ils ont une vision différente de l'Empire, les francophones se heurtent à des obstacles plus importants que leurs concitoyens anglophones lorsqu'ils désirent s'enrôler: les différences linguistiques et culturelles, les préjugés de certains officiers supérieurs de l'armée, la préférence pour les cavaliers de l'Ouest dans certaines unités et, selon La Presse, le fait que les volontaires Canadiens français sont généralement plus petits (c'est pourquoi les critères de taille ont été allégés) et plus nombreux à être mariés plus jeunes, ce qui les disqualifie ${ }^{56}$.

On dénombre environ 55 francophones parmi les membres du premier contingent, le 2nd (Special Service) Battalion du Royal Canadian Regiment of Infantry, qui part pour l'Afrique du Sud le 30 octobre 1899. Une foule de 50000 personnes se rassemble pour saluer leur départ, à Québec, après deux semaines d'intenses préparatifs dirigés par le ministre $\mathrm{F}$. W. Borden et son sous-ministre Louis-Félix Pinault ${ }^{57}$. Le profil des quelque 200 francophones qui sont allés combattre en 
Afrique du Sud ressemble à celui de la majorité des troupes canadiennes, à quelques différences mineures près. Outre qu'ils viennent le plus souvent du Québec et sont surtout catholiques romains, ils sont un peu plus vieux (environ $40 \%$ d'entre eux ont plus de 25 ans) et plus nombreux à être mariés (environ $10 \%$, comparativement à $4 \%$ chez les anglophones). Les francophones laissent aussi derrière eux des emplois de cols blancs, urbains, à faible rémunération, mais ils sont plus nombreux à avoir une expérience militaire (voir le tableau C).

Oscar-Charles Casgrain-Pelletier est l'un de ceux qui sont partis avec le Royal Canadian Regiment. En septembre, Pelletier est informé par le major-général Edward Hutton, officier général commandant de la Milice, qu'il dirigera quatre des huit compagnies régionales, sous le commandement de William Otter ${ }^{58}$. Il commence la guerre au rang de major et commande les compagnies $\mathrm{E}, \mathrm{F}, \mathrm{G}$ et $\mathrm{H}$ pendant presque toute la durée de son service en Afrique du Sud. La compagnie F, l'une des huit compagnies de 125 soldats, représente le district militaire du Québec et compte environ $40 \%$ de francophones. Les francophones de l'Ontario et de l'Acadie sont regroupés dans cette unité ${ }^{59}$. Pelletier est légèrement blessé en février 1900, pendant la bataille de Paardeberg, un tournant de la guerre. Les Canadiens y jouent un rôle important, quoique cette importance ait été exagérée par les Britanniques. Selon un témoignage, «few of this gallant Company of Great Britain's defenders could speak English ${ }^{60}$ ». Leur rôle exact n'est pas bien défini, mais les francophones sont présents ${ }^{61}$.

Un soldat de la compagnie F, Marie-Joseph-Eugène Fiset, s'est rendu célèbre pendant et après la guerre. Fiset, médecin, et le père O'Leary, le prêtre catholique de la compagnie, suscitent l'admiration des Canadiens et des Britanniques 
Tableau C

Les francophones dans les contingents canadiens envoyés en Afrique du Sud*

\begin{tabular}{|c|c|c|}
\hline & 122 francophones** & 56 francophones ou anglophones** \\
\hline Lieu de naissance & $\begin{array}{l}\text { - Québec : } 99 \\
\text { - Extérieur du Québec : } 23 \text {, dont } 1 \\
\text { aux États-Unis, } 3 \text { en France et } 1 \text { à } \\
\text { Saint-Pierre-et-Miquelon }\end{array}$ & $\begin{array}{l}\text { - Québec : } 21 \\
\text { - Extérieur du Québec : } 35\end{array}$ \\
\hline Régiment & $\begin{array}{l}\text { - 2nd (Special Service) Battalion du } \\
\text { Royal Canadian Regiment of Infantry } \\
: 44 \\
\text { - 4th Regiment Canadian Mounted } \\
\text { Rifles : } 31\end{array}$ & $\begin{array}{l}\text { - 2nd (Special Service) Battalion du } \\
\text { Royal Canadian Regiment of Infantry : } \\
13 \\
\text { - } 4 \text { th Regiment Canadian Mounted } \\
\text { Rifles : } 9\end{array}$ \\
\hline Âge & $\begin{array}{l}-17 \text { à } 19 \text { ans : } 1 \\
-20 \text { à } 25 \text { ans : } 74 \\
-26 \text { à } 39 \text { ans : } 46 \\
- \text { Âge inconnu : } 1\end{array}$ & $\begin{array}{l}-17 \text { à } 19 \text { ans : } 2 \\
-20 \text { à } 25 \text { ans : } 37 \\
-26 \text { à } 39 \text { ans : } 10 \\
- \text { Âge inconnu : } 7\end{array}$ \\
\hline État civil & $\begin{array}{l}\text { - Mariés : } 10 \\
\text { - Célibataires : } 107 \\
\text { - État civil inconnu : } 5\end{array}$ & $\begin{array}{l}\text { - Mariés : } 2 \\
\text { - Célibataires : } 47 \\
\text { - État civil inconnu : } 7\end{array}$ \\
\hline Date d'entôlement*** & $\begin{array}{l}\text { - Avril } 1902: 45 \\
\text { - Octobre } 1899: 38\end{array}$ & $\begin{array}{l}\text { - Avril 1902:27 } \\
\text { - Octobre } 1899: 10\end{array}$ \\
\hline Métiers les plus courants & $\begin{array}{l}\text { Soldat : } 15 \\
\text { - Commis : } 10 \\
\text { - Tailleur : } 8 \\
\text { - Journalier : } 6\end{array}$ & $\begin{array}{l}\text { - Joumalier : } 7 \\
\text { - Soldat : } 5 \\
\text { - Commis : } 5\end{array}$ \\
\hline Expérience militaire & $\begin{array}{l}-0 \text { à } 13 \text { ans }: 18 \\
\text { - Un peu (non précisé) }: 77 \\
\text { - Aucune }: 25\end{array}$ & $\begin{array}{l}-0 \text { à } 13 \text { ans : } 5 \\
\text { - Un peu (non précisé) }: 24 \\
\text { - Aucune : } 20\end{array}$ \\
\hline Morts et blessés & $\begin{array}{l}\text { - Tués : } 2 \\
\text { - Morts : } 4 \\
\text { - Blessés : } 1\end{array}$ & $\begin{array}{l}\text { - Tués : } 0 \\
\text { - Morts : } 2 \\
\text { - Blessés : } 1\end{array}$ \\
\hline
\end{tabular}

* Source : Bibliothèque et Archives Canada, documents de Carman Miller, MG31 G36. Cette base de données contient des renseignements sur 5825 des 7368 volontaires canadiens.

** Pour les besoins de notre étude, les 122 soldats dits francophones et les 56 aux noms le plus souvent francophone qui sont dits francophones ou anglophones sont considérés ici comme francophones. Les 178 francophones parmi les 5825 soldars canadiens énumérés dans cette base de données seraient environ 200 si l'on avait pu compter le total des 7368 soldats. La plupart des membres de la force constabulaire d'Afrique du Sud ont disparu. Voir Carman Miller, " A Preliminary Analysis of the Socio-Economic Composition of Canada's South African War Contingents n, Histoire sociale, vol. VIII, n' 16 (1975), p. 219 à 237.

*** Dates les plus courantes 
par leur façon d'aider les blessés sous le tir ennemi. Fiset obtient l'Ordre du Service distingué pour ses efforts et il est mentionné dans les dépêches à trois reprises ${ }^{62}$. Il est fait prisonnier en mai 1900, lorsque, malade, il est abandonné à Heilbron, dans l'État libre d'Orange. Le général boer DeWet aurait été surpris de rencontrer un francophone se battant pour les Britanniques et curieux d'apprendre comment la minorité française était traitée au Canada. Selon un témoignage contemporain, la réponse de Fiset aurait convaincu DeWet que le joug britannique n'était peut-être pas si lourd ${ }^{63}$. Il se peut aussi que sa réponse ait convaincu le général de continuer à se battre encore deux ans. La Canadian Military Gazette qualifie d'exemplaire le parcours militaire de Fiset durant la guerre : major en 1899, il devient lieutenant-colonel en 1901, puis sous-ministre de la Milice et de la Défense de 1906 à 1923. Il est élu député libéral dans la circonscription fédérale de Rimouski avant d'être nommé lieutenant-gouverneur du Québec le 14 décembre 1939. Il a occupé cette fonction jusqu'au $1^{\text {er }}$ octobre 1950.

François-Louis Lessard est peut-être mieux connu des membres francophones des unités canadiennes en Afrique du Sud. Militaire de carrière, formé au Collège militaire royal (CMR), natif de Québec, il commande le Royal Canadian Dragoons. Lorsque la guerre éclate, La Patrie diffuse sa proposition, annoncée publiquement, de diriger les volontaires ${ }^{64}$. Il est invité à commander l'une des unités de fusiliers à cheval du deuxième contingent canadien, qui arrive le 26 mars 1900. Le premier bataillon canadien de fusiliers à cheval, qui compte environ 370 hommes, prend rapidement le nom de Royal Canadian Dragoons ${ }^{65}$. L'un des grands moments de la vie de Lessard se produit en novembre 1900, à Liliefontein, où des Canadiens couvrent des Britanniques poursuivis par un groupe considérable de Boers. Mentionné deux fois dans les dépê- 
ches, Lessard est nommé Compagnon de l'Ordre du Bain pour sa contribution à l'effort de guerre ${ }^{66}$. Tous les témoignages attestent qu'il est un dirigeant populaire, respecté et aimé de ses hommes. Comme le dit l'un d'entre eux, « as a soldier there was none better and we all loved him $^{67} \%$.

Un autre francophone du deuxième contingent, le major J.-A.-G. Hudon, se voit confier la direction de la batterie C du Royal Canadian Field Artillery en Afrique du Sud. Cette unité a vu moins d'action, mais a tout de même participé à la libération de Mafeking en mai 1900. Le lieutenant-colonel T.-L. Boulanger commande le 4th Regiment Canadian Mounted Rifles, qui compte des francophones du Québec et des Maritimes dans les derniers contingents, mais n'a pas l'occasion de participer aux combats. Outre les contingents autorisés par Ottawa, environ 2000 Canadiens servent dans des unités non canadiennes ${ }^{68}$. Philippe-Henri Duperron-Casgrain, diplômé du CMR, est adjudant-général adjoint au quartier général britannique en Afrique du Sud. Le capitaine HenriGustave Joly de Lotbinière, fils d'Henri-Gustave Joly de Lotbinière, premier ministre du Québec et ministre sous Laurier, est sous-directeur de la ligne de Bloemfontein, aux côtés du Canadien le plus connu parmi ceux qui servent en dehors des unités canadiennes, l'ingénieur Édouard Percy Cranwill Girouard.

Né en 1867 à Montréal, Girouard est formé au CMR. Il rejoint les rangs du Royal Engineers comme major en 1889 et participe à une expédition dans la région du Nil en 1897, à l'occasion de laquelle il est mentionné dans les dépêches. Il est directeur des chemins de fer du Soudan de 1896 à 1898 et président de la commission égyptienne des chemins de fer en 1898-1899. Lorsque la guerre éclate en Afrique du Sud, lord Kitchener devient chef d'état-major du commandant lord Roberts et nomme Girouard au poste de directeur des che- 
mins de fer d'Afrique du Sud. Il s'en tire si bien avec le peu de moyens mis à sa disposition qu'il impressionne rapidement son entourage en Afrique du Sud, comme il l'a fait au Soudan ${ }^{69}$. L'historien Thomas Packenham écrit que, durant la marche à travers la colonie de la rivière Orange et du Transvaal, en mai et juin 1900, les soldats britanniques éprouvent beaucoup de difficultés à cause de la destruction des ponts et d'autres infrastructures par les Boers, mais que les réparations ont été grandement facilitées par Girouard, «the man who straightened out these knots - in effect, the chief hero of the advance ». Packenham ajoute que, bien que Girouard n'ait que 33 ans, sa promotion à un si jeune âge est « one of Kitchener's few really successful ideas ${ }^{70} »$. Girouard obtient une très grande reconnaissance pour son travail. Un article paru dans la Canadian Military Gazette de 1907 souligne que : « he is generally acknowledged to be the most brilliant of the many credible graduates of our Royal Military College ${ }^{71}$ ». En 1903, un concours du Montreal Herald le classe au septième rang des "plus grands Canadiens vivants ${ }^{72}$ ».

Les tensions entre francophones et anglophones ne semblent pas avoir touché les soldats canadiens. Ces derniers se rappellent leur voyage au Québec avec grand plaisir et, plus important encore, ils indiquent que, sur le terrain, les relations entre francophones et anglophones ne sont pas problématiques $^{73}$. On fait plus grand cas des tensions entre Canadiens et Britanniques, car beaucoup critiquent les soldats anglais, et plus encore les officiers ${ }^{74}$. La montée d'un sentiment nationaliste canadien au sein des troupes est signalée par le principal spécialiste de la Guerre des Boers, Carman Miller, qui écrit : "Although imperialists had hoped that Canadian participation in a South African war would solidify the formal ties of Empire, many Canadian soldiers returned from the war, not as advocates of imperial integration, but "singing 
their own Canadian war song", determined to fight only under Canadian officers in any future $\operatorname{war}^{75}$.»

\section{L'impact de la guerre}

Les Canadiens français, notamment au début de la guerre, appuient de manière modérée l'intervention canadienne en Afrique du Sud, et certains y participent. Cependant, la mémoire collective n'a retenu que l'opposition à cette guerre. Pourquoi donc ? C'est la façon dont le Canada et le Québec ont évolué au cours du reste du $\mathrm{XX}^{\mathrm{e}}$ siècle, notamment dans trois domaines, qui a déterminé ce qui a été retenu de l'expédition militaire canadienne en Afrique du Sud.

Premièrement, les espoirs nourris par certains pour l'Empire britannique ne se sont pas concrétisés. Le mouvement impérialiste devient plus actif et influent durant la dernière décennie du $\mathrm{XIX}^{\mathrm{e}}$ siècle, ce que révèlent les réactions enthousiastes au Canada devant les demandes de troupes de la part des Britanniques, alors que, à peine quinze ans auparavant, on n'avait guère tergiversé avant de refuser d'envoyer des troupes au Soudan ${ }^{76}$. La plupart des sympathisants sont attirés par l'idée d'un impérialisme ouvert et démocratique faisant la promotion d'une union plus étroite des membres de l'Empire britannique par la coopération économique et militaire et par une transformation politique qui permettra aux dominions d'exercer leur influence sur la politique impériale ${ }^{77}$. Mais d'autres points de vue, partagés par des extrémistes, s'expriment aussi. Certains francophones s'inquiètent des ambitions de ces derniers, qui aspirent à rendre hégémoniques la langue anglaise et le protestantisme ${ }^{78}$. Les rêves des nouveaux impérialistes ne se concrétisent pas, en grande partie à cause des événements d'Afrique du Sud. Le mouvement impérialiste devait être mis à l'épreuve, et la Guerre des Boers en a été l'épreuve malheureuse. Les Uitlanders avaient beau être pri- 
vés de droit de vote au Transvaal, leur plaidoyer de victimes opprimées et sans défense n'était guère convaincant.

Il demeure qu'un engagement militaire pour défendre cette cause, quoiqu'elle n'ait pas suscité l'enthousiasme, a été toléré. Les dirigeants politiques, religieux, intellectuels et militaires francophones ont défendu l'Empire (avec, il est vrai, plus d'enthousiasme que la population canadienne-française en général) et, lorsque leur réaction a été jugée trop tiède, leurs soupçons à l'égard des intentions des Britanniques se sont amplifiés. Un journaliste de l'époque fait remarquer que la presse anglophone ne s'intéresse qu'à l'opposition francophone et il se plaint du fait que la liberté des sujets britanniques de critiquer leurs dirigeants semble réservée aux seuls Anglai $^{79}$. Lord Minto fait également observer en privé que la presse ontarienne traite inégalement les critiques de la guerre : les Britanniques qui contestent ne sont pas considérés comme des traitres, tandis que les Canadiens sont accusés dans ce cas de tiédeur et les Canadiens français, de rébellion ${ }^{80}$.

La Grande-Bretagne n'avait probablement pas besoin de l'aide militaire canadienne pour vaincre les Boers ${ }^{81}$. Londres et les impérialistes canadiens cherchaient avant tout une démonstration de solidarité des membres de l'Empire. Le nombre d'hommes envoyés devient l'objet d'une sorte de compétition entre les dominions, voire un test de loyauté. Robert Philp, premier ministre du Queensland, en Australie, révèle à Laurier le nombre d'hommes fournis par son territoire et le met au défi de faire mieux, tandis que Sam Hughes prévient le chef libéral qu'il serait indigne du Canada d'envoyer moins de soldats que l'Australie ou la Nouvelle-Zélande. Selon lui, cela détruirait le prestige du premier ministre ${ }^{82}$. Compte tenu de sa population, la contribution du Canada (7368 hommes) représente environ un quart de celle de l'Australie (16 415 hommes) et de la Nouvelle-Zélande (6 513 hommes) et un 
sixième de celle de la Grande-Bretagne (355 750 hommes) ${ }^{83}$. Mais les dirigeants britanniques ne se plaignent pas et auraient même plutôt tendance à magnifier la contribution canadienne. Encore une fois, ils ne cherchent pas tant à obtenir une aide militaire qu'un appui politique. Dans cette perspective, la taille des contingents est, pour eux, d'une importance secondaire.

Leur fervent appui à l'Empire a tendance à rendre les Canadiens anglais les plus enthousiastes aveugles aux raisons, légitimes, pour lesquelles les Canadiens français hésitaient à s'enrôler, comme il leur fait oublier le rôle significatif que certains Canadiens français ont joué au sein de l'armée. L'interprétation de la participation du Canada à la guerre pendant et peu après la guerre a tendance à être favorable à la cause britannique et à s'intéresser à la contribution militaire du Canada anglais. L'opposition à la guerre, considérée par la plupart de ces auteurs comme un phénomène exclusivement canadien-français, est pour eux difficile à expliquer et ils considèrent généralement que les responsables politiques y ont accordé trop d'importance ${ }^{84}$. Parmi les francophones, beaucoup se convainquent que les décideurs de Londres veulent, ainsi que les impérialistes canadiens, instaurer un Empire fondé sur l'hégémonie anglo-protestante et insistent de plus en plus pour que la politique canadienne, notamment sur le plan militaire, soit conçue en fonction des intérêts du Canada plutôt que de ceux de la Grande-Bretagne ${ }^{85}$.

Deuxièmement, les Canadiens français n'ont pas joué un rôle important dans la définition de la structure de l'armée régulière professionnelle du Canada. D’une part, l'expérience militaire en Afrique du Sud a enseigné à la milice des leçons importantes au sujet de la guerre moderne, qui ont permis, plus tard, de préparer la Première Guerre mondiale ${ }^{86}$. D'autre part, beaucoup de Canadiens anglais, influencés par les harangues de la presse extrémiste, en sont venus à croire que les 
Canadiens français avaient trahi, n’avaient pas participé et ne devraient donc pas jouer de rôle dans la politique d'aprèsguerre, qu'elle soit d'ordre militaire ou autre. Les tentatives pour inclure des francophones dans l'armée permanente ont échoué $^{87}$. Au lieu de corriger le problème de l'exclusion des Canadiens français de la milice, la guerre n'a fait, à ce chapitre, qu'empirer la situation ${ }^{88}$

À mesure que les Canadiens français réalisent que les critiques canadiens-anglais les plus acerbes considèrent insuffisante leur contribution militaire, ils s'intéressent de moins en moins à toute participation ultérieure ${ }^{89}$. L'évolution de la position de Hutton est, de ce point de vue, sans doute représentative de celle des autres officiers supérieurs. Hutton a essayé de promouvoir le bilinguisme au sein de la milice à la fin du XIX ${ }^{e}$ siècle. Cependant, lorsqu'il estime que l'appui des Canadiens français à la cause de l'Empire en Afrique du Sud est insuffisant, sa tolérance et sa bonne volonté se transforment en haine ${ }^{90}$. L'influence croissante de Sam Hughes donne également à penser que, pour les décideurs canadiens-anglais, la participation des francophones n'est pas une priorité. "Jingo" francophobe parmi les plus acerbes au cours de la guerre en Afrique du Sud, Hughes deviendra, en 1911, ministre conservateur de la Milice et de la Défense. Durant la Première Guerre mondiale, il fera tout ce qu'il peut pour que les francophones se sentent indésirables : il refuse les projets d'unités canadiennes-françaises, engage des anglophones pour recruter au Québec et ne donne pas de rôle important à Lessard et à Pelletier ${ }^{91}$.

La conscription n'est pas vraiment à l'ordre du jour au cours de cette guerre. Même la Grande-Bretagne ne l'a pas imposée. Cependant, les Canadiens français craignent que les critiques à l'endroit de leur participation insuffisante n'amènent les autorités à les contraindre à s'engager dans les con- 
flits à venir. On connait le cas d'une personne qui s'est cachée dans la forêt pendant cinq jours, de crainte d'être enrôlée de force pour combattre en Afrique du Sud ${ }^{22}$. Après la crise de 1917, la question de la conscription sera vue de façon différente ${ }^{93}$.

Troisièmement, les tensions entre anglophones et francophones deviennent de plus en plus un thème de prédilection au sein des deux groupes culturels. Les Canadiens français, pendant la première moitié du siècle, mettront beaucoup l'accent sur l'importance de la survivance. Leur identité est marquée par le catholicisme et le besoin de lutter comme minorité contre les menaces « anglo-saxonnes ${ }^{94} »$. Des dirigeants nationalistes canadiens-français, comme Armand Lavergne, Olivar Asselin, Frederick D. Monk et Bourassa, gagnent en influence grâce à leur dénonciation de la Guerre des Boers ${ }^{95}$. La position de Laurier jouit d'un bon appui à l'époque ${ }^{96}$, mais les commentaires désobligeants des extrémistes canadiensanglais seront rappelés et colportés, avec exagération, par un nombre 'croissant d'historiens nationalistes canadiens-français ${ }^{97}$.

Tout au long du XX $\mathrm{XX}^{\mathrm{e}}$ siècle, beaucoup de questions, de la crise de la conscription au débat sur l'Accord du Lac Meech, ont divisé les Canadiens de part et d'autre de la barrière linguistique. Les francophones et les anglophones ne sont pas d'accord, les radicaux se critiquent les uns les autres et la polarisation s'accentue jusqu'à ce que chacun voit l'autre comme un bloc monolithique aspirant a dominer la politique du pays ${ }^{98}$. Les Canadiens français ont dans un premier temps accepté l'intervention en Afrique et l'ont même modérément appuyée. Cependant, à mesure que la guerre se prolongeait, ils se sont insurgés, non seulement contre la politique britannique, mais plus encore contre les attaques répétées des extrémistes de la presse ontarienne, qui les accusaient de trahison. Ces atta- 
ques, qui auraient peut-être été plus faciles à supporter si les Canadiens français avaient réellement trahi, visaient les francophones qui s'opposaient à la guerre et refusaient d'y participer. Dans ce contexte, certains Canadiens français sont devenus rétroactivement des opposants à la guerre, perpétuant volontiers le mythe selon lequel ils l'avaient toujours été. Ceux qui ont appuyé la guerre et y ont participé, eux, sont tombés dans l'oubli.

À mesure que la présence britannique dans la vie publique du Canada diminue, les historiens canadiens-anglais remettent de plus en plus en question l'hypothèse selon laquelle les actions des autorités britanniques étaient aussi désintéressées et admirables qu'elles le prétendaient. L'hésitation des francophones à s'associer à la croisade impériale est mieux comprise, mais on continue néanmoins de s'intéresser davantage à leur opposition à la guerre ${ }^{99}$. Certains historiens expliquent plus en détail la réaction des Canadiens français ${ }^{100}$, mais la plupart des historiens nationalistes franco-québécois parlent de la guerre - lorsqu'ils le font - comme d'une aventure impérialiste britannique à laquelle les Canadiens français se sont fortement opposés parce qu'ils savaient qu'elle créait un dangereux précédent qui risquait d'entraîner le Canada dans d'autres guerres impérialistes, plus sanglantes encore ${ }^{101}$.

\section{$* * *$}

Parmi les interventions militaires du Canada au $\mathrm{XX}^{\mathrm{e}}$ siècle, celle de l'Afrique du Sud est la plus distante, tant sur le plan géographique que chronologique. Des événements ultérieurs ont facilité la tâche de ceux qui ont préféré se rappeler de l'opposition à la participation du pays à cette guerre et oublier que les Canadiens français, à l'origine, l'ont probablement en majorité appuyée, et que certains d'entre eux ont même servi, avec beaucoup de succès, en Afrique du Sud. 
Cela permet de jeter un éclairage neuf sur l'échec du mouvement impérialiste, les problèmes d'équilibre linguistique au sein de l'armée canadienne, l'opposition des Canadiens français à la conscription, ainsi que les relations souvent tendues entre francophones et anglophones pendant le reste du $\mathrm{XX}^{\mathrm{e}}$ siècle.

Traduction : Ministère de la Défense nationale

\section{NOTES}

' Je désire remercier tous ceux qui m'ont aidé à améliorer les versions antérieures de ce texte, notamment Marie Taillon, Yves Bégin, Damien-Claude Bélanger, Carman Miller, Jean-Guy Pelletier, Cameron Pulsifer, Dominique FoisyGeoffroy, ainsi que les évaluateurs anonymes de mon texte.

${ }^{2}$ " 100 Québécois qui ont fait le $20^{\mathrm{c}}$ siècle », L'Actualité, février 1999. Voir aussi « 25 jeunes de 25 ans construisent l'avenir dans 25 coins du monde», L'Actualité, 15 septembre 2000 ; Roch Legault, Une élite en déroute : Les militaires canadiens après la Conquête, Montréal, Athéna, 2002, p. 163.

${ }^{3}$ Jacques Mathieu et Jacques Lacoursière, Les mémoires québécoises, Sainte-Foy, Presses de l'Université Laval, 1991, p. 328 à 341.

${ }^{4}$ Matt Hartley et Joanna Smith, "Legendary Vandoos "do the job right"”, The Globe and Mail, 16 juillet 2007 ; Lisa-Marie Gervais, «Le risque québécois », Le Devoir, 16 juillet 2007 ; sondages Léger Marketing d'octobre 2001 et de juin 2007, www.legermarketing.com, consultés le 17 juillet 2007. À la question "Au moment de débuter les frappes sur l'Afghanistan, le Canada a décidé d'accorder un appui militaire aux États-Unis. Est-ce que vous appuyez FORTEMENT, EN PARTIE ou PAS DU TOUT cet appui militaire du Canada ? ", posée en octobre 2001, 36,3\% des Québécois ont répondu " fortement », $42,1 \%$, « en partie », et $17,0 \%$ « pas du tout ».

${ }^{5}$ Un sondage fait état du soutien de $53 \%$ des répondants au Québec en février 1991, peu après le déclenchement de la guerre. Voir Jocelyn Coulon, La dernière croisade : La guerre du golfe et le rôle caché du Canada, Montréal, Méridien, 1992, p. 109. 
${ }^{6}$ Selon un sondage Gallup effectué au cours de la crise, les Canadiens soutiennent la position du gouvernement à $85 \%$. La population du Québec constituant, en 1971, près de $28 \%$ de l'ensemble de la population canadienne, il est ainsi presque certain qu'au moins $50 \%$ des Québécois ont appuyé la Loi des mesures de guerre. Selon un autre sondage, publié dans Le Devoir le 19 octobre 1970, soit trois jours après l'entrée en vigueur de la loi et deux jours après la découverte du corps de Pierre Laporte, la priorité de $55 \%$ des Québécois était de "maintenir l'ordre». Voir Richard Gwyn, The Northern Magus, Markham, McClelland and Stewart-Paper Jacks, 1981, p. 133 ; Bulletin d'histoire politique, vol. 11, n 1 (automne 2002), consacré à « La mémoire d'octobre : art et culture "; Manon Leroux, Les silences d'octobre: les discours des acteurs de la Crise de 1970, Montréal, VLB, 2002,p. 125, dans lequel l'auteur fait remarquer que Trudeau et Bourassa ont tous deux affirmé plus d'une fois qu'ils jouissaient du soutien de la population, tandis que les opposants invoquaient rarement l'opinion publique.

${ }^{7}$ John MacFarlane, «French-Canadian Views of Collective Security, 19451950 », dans Yves Tremblay, L'bistoire militaire canadienne depuis le XVII' siècle, Ottawa, Ministère de la Défense nationale, 2001, p. 584 et 585.

${ }^{8}$ John MacFarlane, Ernest Lapointe and Québec's Influence on Canadian Foreign Policy, Toronto, University of Toronto Press, 1999, p. 146 à 150. Voir aussi Bulletin d'bistoire politique, vol. $3, \mathrm{n}^{\text {os }} 3$ et 4 (printemps-été 1995), consacré à « La participation des Canadiens français à la Deuxième Guerre mondiale : mythes et réalités ». Au sujet de la Première Guerre mondiale, voir Paul-André Linteau, René Durocher et Jean-Claude Robert, Histoire du Québec contemporain : de la Confédération à la Crise (1867-1929), Montréal, Boréal, 1979, p. 598 à 600 ;. Desmond Morton et J. L. Granatstein, Canada and the Two World Wars, Toronto, Key Porter, 2003, p. 17.

${ }^{9}$ Bibliothèque et Archives Canada, RG 24, vol. 4516-8. Y est incluse l'« Enquête tenue devant le coroner pour le district de Québec le 8 avril et les jours suivants sur les causes de la mort de Honoré Bergeron, Alexandre Bussière, Georges Demeule et Édouard Tremblay ». Le chef de police y fait remarquer que plus de 2500 personnes avaient demandé une protection supplémentaire durant la fin de semaine. Voir Jean Provencher, Québec sous la loi des mesures de guerre, 1918, Montréal, Boréal, 1971, p. 100.

${ }^{10}$ Milner à lord Roberts, 6 juin 1900, cité dans Thomas Packenham, The Boer War, Londres, Weidenfield and Nicholson, 1979, p. 115. Voir aussi Carman Miller, Painting the Map Red: Canada and the South African War, 1899-1902, 
Ottawa, Montréal et Kingston, Musée canadien de la guerre et McGill-Queen's University Press, 1993, p. 14.

${ }^{11}$ Documents parlementaires, Ministère de la Milice et de la Défense, $\mathrm{n}^{\circ}$ 20,1900, p. 11 à $16: \mathrm{J}$. Chamberlain à sir W. MacGregor, Lagos, 21 juillet $1899 ; \mathrm{J}$. Chamberlain au haut commissaire C. Mitchell, États fédérés de Malaisie, 18 juillet 1899 .

${ }^{12}$ Mordechai Tamarkin, « Milner, The Cape Afrikaners, and the Outbreak of the South African War: From a Point of Return to the Dead End», The Journal of Imperial and Commonwealth History, vol. 25, $\mathrm{n}^{\circ} 3$ (septembre 1997), p. 393 et 410 .

${ }^{13}$ M. van Wyk-Smith, Drummer Hodge: the Poetry of the Anglo-Boer War, 18991902, Oxford, Clarendon Press, 1978, p. xi, 12, 22 et 35.

${ }^{14}$ Laurier à Ernest Pacaud, 9 juillet 1897 . Il y fait remarquer qu'il est fier de ses origines françaises, mais aussi profondément attaché aux institutions britanniques et que, à titre de premier ministre, il est tenu de manifester sa loyauté. Voir Jean-Guy Pelletier, Les Canadiens français et la Guerre des Boers, mémoire de M.A. (histoire) Université Laval, 1963, p. 11.

${ }^{15}$ Bibliothèque et Archives Canada, documents de Minto, MG27 II B1, Minto à Chamberlain, 25 juillet 1899. Minto était sensible aux préoccupations de Laurier et y souscrivait parfois. En mai 1899, il déclare à Laurier qu'il s'oppose à l'intervention du Canada en Afrique du Sud et, en juillet, à Chamberlain que : « the French-Canadians are very loyal, but you must remember they are French ». Le 23 septembre, il avertit Chamberlain de ne pas pousser Laurier, car l'opposition au Québec est forte. Voir Aileen Elizabeth Barker, Laurier, French Canada and the Boer War, Vancouver, University of British Columbia, 1961, p. 34, 66 ; Miller, Painting the Map Red, p. 33 à 37.

${ }^{16}$ Bibliothèque et Archives Canada, documents de Laurier, C-769, 37912, Révérend L. Tremblay, curé de St-Félicien, à Laurier, 10 octobre 1899 : « Pour l'amour de Dieu faites tout ce qui est possible pour ne pas aider Chamberlain dans son injuste guerre contre le Transvaal. » Réponse de Laurier le 15 octobre $1899,38097$.

${ }^{17}$ Chambre des Communes, Débats, 23 avril 1902, p. 3352, et 12 mars 1901, p. 1336. Ce qui était en jeu dans la guerre, selon Laurier, c'était de savoir qui devrait gouverner, les Britanniques ou les Hollandais à moitié barbares.

${ }^{18} \mathrm{Haut}$ Commissariat du Canada à Londres : lord Strathcona propose d'abord de parrainer une unité de cavaliers le 31 décembre 1899. Au sujet de la force 
constabulaire d'Afrique du Sud voir Miller, Painting the Map Red, p. 368 à 390, et « The Unhappy Warriors: Conflict and Nationality among Canadian Troops during the South African War ", Journal of Imperial and Commonwealth History, vol. 23, n" 1 (1995), p. 77-104. Le Cabinet finit par accepter malgré lui d'envoyer la dernière troupe de 2000 hommes, en raison des pressions publiques et de la demande de Chamberlain datée du 18 mars 1902, qui fait remarquer que la Nouvelle-Zélande a fait cadeau de 1000 hommes de plus.

${ }^{19}$ Bibliothèque et Archives Canada, documents de Laurier, C-772, 40781, Laurier à Strathcona, 31 janvier 1900, Laurier à C.A. Semlin, premier ministre de la C.-B., 13 février 1900. Bien qu'il accepte la formation du Strathcona's Horse, il exprime sa crainte de « complications » et préfère que ce soit Chamberlain qui prenne la décision.

21) Bibliothèque et Archives Canada, documents de Minto, vol. 128, dossier 233, réponse de Minto à Chamberlain, 22 février 1900, et demande de Chamberlain à Minto, 21 février 1900. Les Britanniques demandent aux Canadiens d'approuver l'envoi du deuxième bataillon canadien de fusilliers à cheval (BCFC) en février 1901 et en novembre 1901 : Bibliothèque et Archives Canada, RG7 G21, vol. 129, dossier 233, Chamberlain à Minto, 15 novembre 1901 ; Minto à Chamberlain, 25 mars 1902. Laurier accepte tout de même d'envoyer quatre autres contingents (les $3^{\mathrm{e}}, 4^{\mathrm{e}}, 5^{\mathrm{e}}$ et $6^{\mathrm{e}} \mathrm{BCFC}$ ), peut-être influencé par le rappel de Minto que tout refus pourrait être interprété comme " a want of enthusiasm for the imperial cause » lors de la prochaine Conférence impériale, prévue pour juin 1902. Minto à Laurier, 19 mars 1902 : voir Miller, Painting the Map Red, p. 414.

${ }^{21}$ La Presse, 27 octobre 1899, p. 4. Voir aussi Victor Gagné à sa mère, 2 décembre 1900, dans Le Courrier du Canada, 5 février 1901, reproduit dans Pelletier, Les Canadiens français et la Guerre des Boers, p. 33. Au sujet du prisonnier, voir Miller, Painting the Map Red, p. 237.

${ }^{22}$ Bibliothèque et Archives Canada, documents de Laurier, C-769, 38426, T. Macauley à Laurier, 28 octobre 1899. Voir aussi C-771, 39996, T. Robson, 19 décembre 1899. C-770, 38884, P. Thompson, 13 novembre 1899. Selon George M. Grant, la position des impérialistes refléterait l'opinion unanime du Canada anglais. Bibliothèque et Archives Canada, documents de Laurier, C-771, 39908, George Grant à Laurier, 17 décembre 1899. Laurier répète qu'il n'a fait que ce que lui a demandé le Colonial War Office : Bibliothèque et Archives Canada, documents de Denison, vol. 9 et 10, Laurier à Denison, 14 octobre 1899. Hutton estime également qu'il existe une " détermination commune » à tout le Canada de maintenir à tout prix l'intégrité de l'empire : Rapport du 
ministère de la Milice et de la Défense pour l'année se terminant en décembre 1898, Documents parlementaires, $\mathrm{n}^{\circ}$ 19, 1899, p. 40.

${ }^{23}$ Bibliothèque et Archives Canada, documents de Denison, vol. 9 et 10 , Laurier à Denison, 6 décembre 1899 ; et documents de Laurier, C-770, 38558, Denison à Laurier, $1^{\text {er }}$ novembre 1899.

${ }^{24}$ Mail and Empire, 5 octobre 1899. Copie dans les documents de Laurier, C769. Les pressions sont venues du Montreal Daily Star, du Toronto News et du World. Voir Carman Miller, "English-Canadian opposition to the South African War as seen through the Press ", Canadian Historical Review, vol. LV, ${ }^{\circ}$ 4 (décembre 1974), p. 432-433.

${ }^{25}$ Bibliothèque et Archives Canada, documents de Laurier, C-771, 40611-13, lettres anonymes, 1899 ; on se demande si les Canadiens français appuieraient les Britanniques. Documents de Laurier, C-772, 41379, 23 janvier 1900. Des fermiers, des ouvriers, des membres du clergé protestant et des Canadiens d'origine irlandaise et allemande étaient parmi les dissidents. Voir Miller, Painting the Map Red, p. 22.

${ }^{26}$ Bibliothèque et Archives Canada, RG7 G21, vol. 126 à 128, dossiers numérotés du gouverneur général, 1899-1902, Chamberlain à Minto, 13 octobre 1899. Chamberlain a également accepté des troupes canadiennes le 3 octobre avant qu'on les lui offre : Documents parlementaires, 35a, 1901-1903. Chamberlain déclare plus tard que l'aide fournie était l'expression d'un sentiment croissant d'unité et de solidarité dans l'Empire. Chamberlain à Minto, 15 novembre 1899, Documents parlementaires, ministère de la Milice et de la Défense, $\mathrm{n}^{\circ} 20,1900$, p. 39. Chamberlain et Salisbury mettent l'accent sur les progrès obtenus en matière d'unité impériale : Bibliothèque et Archives Canada, documents de Denison, MG 29 E29, vol. 9 et 10, Denison à Chamberlain, 17 mars 1900, Chamberlain à Denison, 9 mai 1900, Salisbury à Denison, 2 avril 1900. Bibliothèque et Archives Canada, documents de Laurier, C-769, 37824-6, anglophone anonyme, 4 octobre 1899. Voir aussi p. 38016, W. Merritt, United Empire Loyalist Association, 13 octobre 1899 ; et p. 37999, B. Reed, 13 octobre 1899.

${ }^{27}$ Bibliothèque et Archives Canada, documents de Laurier, C-769, 38054-7, J.S. Willison, du Globe, à Laurier, 14 octobre 1899.

${ }^{28}$ Tarte à Castell Hopkins, 1899, cité dans O.D. Skelton, Life and Letters of sir Wilfrid Laurier, vol. II, Toronto, Oxford University Press, 1921, p. 99. 
${ }^{29}$ Biblothèque et Archives Canada, documents de Laurier, C-769, 38045, interlocuteur anonyme à Laurier, 13 octobre 1899. Voir aussi Miller, Painting the Map Red, p. 28 et 29 ; et van Wyk Smith, Drummer Hodge, p. 251.

${ }^{30}$ Gaston P. Labat, dir., Le livre d'or / The Golden Book of the Canadian Contingents in South Africa, Montréal, [s.é.], p. 1 à 13 ; Jean-Guy Pelletier, "Qu'allions-nous faire dans cette galère ? La Guerre des Boers ", Cap-aux-Diamants, $n^{\circ} 48$ (hiver 1997), p. 30-33.

${ }^{31}$ Louis Fréchette, cité dans Labat, Le livre d'or, p. vi-vii.

${ }^{32}$ Dandurand à Laurier, 3 novembre 1899, dans Marcel Hamelin, dir., Les mémoires du sénateur Raoul Dandurand, 1861-1942, [Québec], Presses de l'Université Laval, 1967, p. 71 ; concernant son appui (18 décembre 1899) : Miller, Painting the Map Red, p. 155.

${ }^{33}$ Cité dans Labat, Le livre d'or, p. 3, 7 et 23.

${ }^{34}$ Bibliothèque et Archives Canada, documents de Laurier, C-769, 38058, C. Fitzpatrick, avocat à Québec, à Laurier, 14 octobre 1899. Borden et le ministre de l'Agriculture Sydney Fisher rappellent souvent les 7,5 millions de dollars de biens canadiens achetés par le War Office : Miller, Painting the Map Red, p. 425-427.

${ }^{35}$ Bibliothèque et Archives Canada, documents de Laurier, C-769, 38301, Pacaud, maire de Sorel, à Laurier, 24 octobre 1899 : il se plaint que le Montreal Daily Star n'a pas publié sa réponse approuvant la décision d'Ottawa, alors que le quotidien a seulement publié les lettres de francophones critiquant la participation.

${ }^{36}$ Jules-Paul Tardivel, rédacteur en chef de La Vérité, propose que le Québec quitte la Confédération. Voir Pelletier, «Les Canadiens-français», p. i. Un citoyen accepte d'appuyer la Grande-Bretagne, mais il aurait préféré qu'on n'envoie pas de troupes canadiennes : documents de Laurier, C-769, 38235 ; J.E. Marcile à Laurier, 19 octobre 1899.

${ }^{37}$ Bibliothèque et Archives Canada, documents de Laurier, C-771, 39998, C. Aubin à Laurier, 19 décembre 1899. Voir aussi C-770, 39344, J. Legris à Laurier, 28 novembre 1899.

${ }^{38}$ Bibliothèque et Archives Canada, documents de Bourassa, MG 27 II E1, M-722, Bourassa à J.-P. Tardivel, 14 août 1899 : « je crois pouvoir affirmer que les Canadiens français sont plus respectés à Ottawa qu'ils ne l'ont jamais été $[\ldots]$ le nombre de ceux qui vous regardent comme d'une race inférieure diminue beaucoup ». 
${ }^{39}$ Bibliothèque et Archives Canada, documents de Bourassa, M-722, Bourassa à Laurier, 18 octobre 1899. En privé, il parle de la faiblesse de Laurier : 27 octobre 1899.

${ }^{40}$ Bibliothèque et Archives Canada, documents de Laurier, C-769, 38301, E. Pacaud à Laurier, 24 octobre 1899.

${ }^{41}$ Bibliothèque et Archives Canada, documents de Joseph-Israël Tarte, MG 27 II D 16, vol. 5 à 7. Copies de nombreux articles, dont ceux du Toronto News, 14 octobre 1899. Voir aussi La Patrie, 17 octobre 1899, p. 1.

${ }^{42}$ La Presse, 6 décembre 1899, p. 1. Voir aussi les références à une nation héroïque, La Presse, 11 décembre 1899, p. 4.

${ }^{43}$ La Presse, 14 septembre 1899, p. 4. Concernant les références à l'opposition en Europe, voir La Presse, 29 novembre 1899, p. 5, et 12 décembre 1899, p. 4.

${ }^{44}$ La Presse, 30 septembre 1899, p. 5. Voir aussi un article décrivant une résolution du Club libéral en faveur de l'envoi de troupes, mais précisant également que l'indépendance du Canada est le rêve de tous les vrais patriotes : $\mathrm{La}$ Presse, 19 octobre 1899. Les « jingos » (patriotards), selon le dictionnaire $O x$ ford, étaient des patriotes chauvins, partisans de la politique belliqueuse défendue par le premier ministre anglais Benjamin Disraeli en 1878.

${ }^{45}$ La Presse, 14 octobre 1899, p. $10 ; 2$ novembre 1899, p. 4.

${ }^{46}$ Carman Miller, Canada's Little War: Fighting for the British Empire in Southern Africa, 1899-1902, Toronto, Lorimer, 2003, p. 47 à 54.

${ }^{47}$ Chambre des Communes, Débats, 13 mars 1900, p. 1850. En faveur : Angers, Bourassa, Chauvin, Dugas, Éthier, Legris, Marcil, Marcotte, Monet, Morin. Le Québec était doté de 65 sièges à la Chambre des communes, dont certains étaient occupés par des anglophones, tandis que des francophones d'autres régions du pays s'ajoutaient au nombre de Canadiens français à la Chambre.

${ }^{48}$ Bibliothèque et Archives Canada, documents de Laurier, C-774, 43645, C. Warman à Laurier, 21 mars 1900. Voir aussi Bourassa citant Tarte dans The Canadian Annual Review, 1901, p. 305.

${ }^{49}$ Bibliothèque et Archives Canada, documents de Bourassa, M-721, Goldwyn Smith à Bourassa, 8 février 1900 : il laisse entendre que Laurier est trop impérialiste et aurait perdu si Tupper n'avait pas été pire. Selon William Lyon Mackenzie King, « the Liberals feel that Mr. Tarte has been the heavy load to carry in Ontario ». King, Journal, 13 novembre 1901. Le 8 novembre 1900, W.D. Gregory dit à Bourassa que les Libéraux ont perdu en Ontario, non pas à cause de la guerre, mais de la dette. Documents de Bourassa, M-721. 
${ }^{50}$ Norman Penlington, " Ontario's Contribution to the South African War ", Ontario History, vol. XLII, $\mathrm{n}^{\circ} 4$ (1950), p. 175. Bien qu'ils aient perdu six sièges en Ontario (remportant 37 sièges, contre 55 pour les conservateurs), les libéraux remportent 133 sièges en tout (contre 80 pour les conservateurs). L'Ontario est la seule province qui n'a pas voté libéral et où les libéraux n'ont pas, augmenté le nombre de sièges qu'ils détiennent depuis 1896, exception faite de la Colombie-Britannique, où ils détiennent encore quatre sièges sur six.

${ }^{51}$ Bibliothèque et Archives Canada, documents de Laurier, C-772, 40950-70, sir H. Devilliers à Laurier, 9 janvier 1900. Réponse de Minto, après que Laurier lui a montré la lettre, dans ibid., C-773, 42219, Minto à Laurier, 9 février 1900 : " whatever may be the right or the wrong of the war, the influence of large financiers has played too great a part ... and a powerful section of the press has unscrupulously supported them $»$.

${ }^{52}$ Bibliothèque et Archives Canada, documents de Bourassa, M-721, Bourassa à G. Smith, 17 mai 1900.

${ }^{53}$ Bibliothèque et Archives Canada, documents de Bourassa, M -721, Bourassa à Gregory, 15 mars 1901. Bourassa ne cesse de répéter que, tout en s'opposant à tous les précédents donnant lieu à des engagements envers les guerres impériales, il reste fidèle : Chambre des Communes, Débats, 13 février 1900 , p. 375.

${ }^{54}$ Chambre des Communes, Débats, 12 mars 1901, p. 1306. Il ajoute que la conviction que l'action n'est pas un précédent est vitale et que « le cancer de l'argent a dévoré la société britannique » : p. 1290-1326. Bourassa rappelle que la plupart des Canadiens français sont opposés à la guerre : Chambre des Communes, Débats, 23 avril 1902, p. 3351. Le $1^{\text {er }}$ août 1900, G. Maynard dit également à Laurier que la participation à la guerre n'a pas la faveur des Canadiens français, qui « excusent le gouvernement dont la main a été forcée par l'élément anglais et les circonstances ». Voir Barker, « Laurier», p. 205.

${ }^{55}$ T.G. Marquis désigne du terme d' "Anglo-saxons » les Royal Canadian Dragoons dirigés par Lessard. Erreur notée dans Brian A. Reid, Our Little Army in the Fields: the Canadians in South Africa, 1899-1902, St Catharines, Vanwell Publishing, 1996, p. 100.

${ }^{56}$ Desmond Morton, « Le Canada français et la milice canadienne, 1868-1914», dans Jean-Yves Gravel, dir., Le Québec et la guerre, Montréal, Boréal, 1974, p. 23-37 ; Jean Pariseau et Serge Bernier, Les Canadiens français et le bilinguisme dans les Forces armées canadiennes, tome 1, Ottawa, Ministère des Approvisionnements et Services, 1987, p. 27, 53 ; Desmond Morton, «The Place of French 
Canadians in the Canadian Militia, 1867-1914», Présentation à la Commission royale sur le bilinguisme et le biculturalisme, 1967. Concernant la préférence pour les cavaliers de l'Ouest, voir Kyle McIntyre, "Sons of Good Western Stock... », Histoire militaire canadienne, vol. 6, $\mathrm{n}^{\circ} 1$ (printemps 1997), p. 84-91; La Presse, 25 octobre 1899, p. 1, 7, 9. Dans « La Guerre des Boers », Pelletier fait valoir que le manque d'enthousiasme des Canadiens français est la seule raison pour laquelle les recruteurs ont été contraints de choisir des hommes plus petits.

${ }^{57}$ Miller, Painting the Map Red, p. 55 à 58.

${ }^{58}$ Hutton à Pelletier, 5 septembre 1899, dans col. Oscar C. Pelletier, Mémoires, souvenirs de famille et récits, Québec, [s.é.], 1940, p. 306-308. Voir Michel Litalien, «La carrière militaire du colonel Oscar Pelletier, » dans Roch Legault, dir., Le Leadership militaire canadien-français, Toronto, Dundurn, 2007, p. 181-206.

${ }^{59}$ Dans « La Guerre des Boers » et dans Les Canadiens français et la Guerre des Boers, Pelletier parle de 40 Canadiens français sur 123 hommes dans la Compagnie F. La Patrie du 30 octobre 1899 parle de 60 francophones sur 123 hommes, tandis que, selon la base de données de Miller, il y aurait eu 44 francophones et 13 francophones ou anglophones.

${ }^{60}$ Miller, Painting the Map Red, p. 111, citant Louis Creswicke, South Africa and the Transvaal War. Voir aussi Desmond Morton, Canada at Paardeberg, Ottawa, Musée canadien de la guerre, 1986, p. 14.

${ }^{61}$ Musée canadien de la guerre, documents d'Otter, vol. 102. Dans son journal, il parle, le 27 février 1900, d'une retraite générale, exception faite des Compagnies $\mathrm{G}$ et $\mathrm{H}$, mais, dans ses notes de conférence pour le Collège militaire royal ("The Battle of Paardeberg»), il parle également des francophones de la Compagnie F.

${ }^{62}$ Musée canadien de la guerre, documents d'Otter, vol. 102, "Battle of Paardeberg ».

${ }^{63}$ W. Sanford Evans, The Canadian Contingents and Canadian Imperialism: a Story and a Study, Ottawa, Eugene G. Ursual, [1995], p. 242.

${ }^{64}$ La Patrie, 14 octobre 1899, p. 13 à 15. Voir John MacFarlane, «La force permanente du major-général F.-L. Lessard », dans Legault, dir., Le Leadership militaire canadien-français, p. 207 à 234. Voir aussi John MacFarlane, « The Right Stuff? Evaluating the Performance of Lt-Col F.-L. Lessard in South Africa and his Failure to Receive a Senior Command Position with the CEF in 1914 », Histoire militaire canadienne, vol. 8, nº 3 (1999), p. 48 à 58. 
${ }^{65}$ Brereton Greenhous, Dragoon: the Centennial History of the Royal Canadian Dragoons, 1883-1983, Belleville, Guild of the Royal Canadian Dragoons, 1983, p. 87. Voir aussi F.-L. Lessard, "Reminiscences of the South African War", The Goat [bulletin des Royal Canadian Dragoons], 1925.

${ }^{66}$ Direction de l'histoire et du patrimoine, Lessard, 000.9, (D106), J.F. Cummins, " A Distinguished Canadian Cavalry Officer Major-General F.-L. Lessard, CB », dans Revue canadienne de défense, vol. 3, n 2 (janvier 1926), p. 128 à 131 .

${ }^{67}$ Bibliothèque et Archives Canada, documents d'Albert. E. Hilder, MG 30 E30, "Comrades All: A Narrative of the South African War». Hutton à Minto, 30 octobre 1900 ; Greenhous, Dragoons, p. 136 à 144 ; Reid, Our Little Army, p. 41. Citation de Larry Worthington, "The Spur and the Sprocket": the Story of the Royal Canadian Dragoons, Oromocto, Royal Canadian Dragoons, 1967.

${ }^{(18}$ Reid parle de plus de 2000 (Our Little Army, p. 152); Miller estime qu'au moins 400 hommes ont rejoint les rangs des forces régulières et irrégulières britanniques (Painting the Map Red, p. 445).

(9) Bibliothèque et Archives Canada, documents de Désiré Girouard, MG 30 E313, vol. 6 : article sur Girouard intitulé « Men you know in South Africa », 14 février 1903.

${ }^{70}$ Packenham, The Boer War, p. 422. Voir aussi Reid, Our Little Army, p. 153.

${ }^{71}$ Bibliothèque et Archives Canada, documents de Girouard, vol. 6 : Canadian Military Gazette, 12 juillet 1907. Voir aussi « Girouard feared not Kitchener» : en Égypte, lorsqu'on lui dit de se dépêcher, Girouard répond qu'il ne peut rien sans approvisionnements ; il est remplacé pendant une semaine, puis rétabli après confirmation de ses dires : Ottawa Journal, 3 juillet 1907.

${ }^{72}$ Bibliothèque et Archives Canada, documents de Girouard, vol. 5. «Sir Wilfrid, Strathcona, sir Charles ». Les lecteurs qui ont voté au concours des grands hommes vivants du Canada donnent premier Laurier, deuxième Strathcona, troisième Tupper, quatrième le romancier Gilbert Parker, cinquième William Van Horne, sixième Edward Blake et septième Percy Girouard. Voir d'autres articles sur d'autres concours dans le Montreal Daily Star, 14 octobre 1903. Après la guerre, Girouard reste dans la région, où il devient commissaire aux chemins de fer pour la Transvaal and Orange River Colony (19021904), avant de devenir haut commissaire du Protectorat du Nigeria du Nord (1907-1908), puis gouverneur du Nigeria du Nord (1908-1909). Il est ensuite nommé gouverneur et commandant en chef du Protectorat de l'Afrique 
orientale (1909-1912). Au cours de la Première Guerre mondiale, il fait office de directeur général de l'approvisionnement en munitions pour le British War Office. Il meurt en 1932, à Londres.

${ }^{73}$ Bibliothèque et Archives Canada, documents de Heron, MG 30 E355, vol. 1. Voir aussi : Bibliothèque et Archives Canada, documents de Hilder; et Morrison, With the Guns, p. 18. W. Hart-McHarg, From Quebec to Pretoria, Toronto, Briggs, 1901, p. 51. Seulement quelques soldats racontaient que l'envoi de troupes était moins enthousiaste au Québec. Selon l'un d'entre eux, " we might have been in a strange country ». Lorsque Otter fait état de problèmes d'adaptation mineurs à l'esprit de corps du premier contingent et de jalousies sectaires entre les troupes, il semble parler des soldats de Toronto plus que des Canadiens français : Musée canadien de la guerre, documents d'Otter, vol. 102.

${ }^{74}$ Bibliothèque et Archives Canada, documents de Minto, vol. 16, Minto à Lansdowne, 12 avril 1900 : commentaires sur la critique croissante des officiers britanniques. Voir aussi Bibliothèque et Archives Canada, documents de George Smith, MG 29E 100, 12 juillet 1900 ; documents de Bourassa, vol. 721, Bourassa à Gregory, 15 mars 1901 : il y confie que les volontaires auraient fait des remarques désobligeantes au sujet d'officiers britanniques et que "Sam Hughes himself admitted to me that the farm burning was a useless and even obnoxious tactic and that it had to be stopped $»$.

${ }^{75}$ Miller, "Unhappy Warriors », p. 99. Voir aussi Reid, Our Little Army, p. 172 ; et E.W.B. Morrison, With the Guns in South Africa, Ottawa, Eugene Ursual, Ottawa, 1995 [1901], p. 20.

${ }^{76}$ Concernant le Soudan, voir Serge Bernier, Le Patrimoine militaire canadien, Montréal, Art Global, 2000, p. 88.

${ }^{77}$ Carl Berger, The Sense of Power: Studies in the Ideas of Canadian Imperialism, 1867-1914, Toronto, University of Toronto Press, 1970, p. 3, 5, 134, 152. C'est une force politique, économique, sociale et spirituelle qui prend de l'ampleur au moment des célébrations du jubilé de diamants de la reine Victoria, en 1897. Voir aussi Robert Page, "The Boer War and Canadian Imperialism ", Ottawa, Société historique du Canada, 1987, p. 4-8 ; Miller, Painting the Map Red, p. 4-7. La British Empire League (anciennement Imperial Federation League), quoiqu'elle ne comptât jamais beaucoup de membres et ne peut être considérée comme étant représentative du Canada anglais dans son ensemble, est le groupe le plus organisé. 
${ }^{78}$ Joseph Chamberlain évoque, devant un auditoire de Toronto en 1887, le destin grandiose de la " race anglo-saxonne ", appelée à être une force dominante dans l'histoire du monde. Cité dans Robert Craig Brown et Ramsay Cook, Canada 1896-1921: a Nation Transformed, Toronto, McClelland and Stewart, 1974, p. 26.

${ }^{79}$ La Presse, 15 novembre 1899, p. 4.

${ }^{80}$ Minto, dans une lettre adressée à son frère en juin 1900, dans Page, Imperialism, p. 20.

${ }^{81}$ À Denison, qui déclare que, après avoir envoyé 1000 hommes, « we are involved, so that in case of need we cannot abandon them, but must send more », Laurier répond que « if England cannot fight that little war [...] all my conceptions of England's power were wrong ». Bibliothèque et Archives Canada, documents de Denison, vol. 9 et 10, Laurier à Denison, 6 décembre 1899. Voir aussi les documents de Laurier, C-770, 38558, Denison à Laurier, $1^{\text {er }}$ novembre 1899.

${ }^{82}$ Bibliothèque et Archives Canada, documents de Laurier, C-772, 41905, Philp à Laurier, 2 février 1900 ; C-769, 37878-9, Sam Hughes à Laurier, 6 octobre 1899.

${ }^{83}$ Page, Imperialism, p. 15. L'Afrique du Sud envoie 50000 hommes, l'Inde et Ceylan, 500. Voir aussi John Stirling, The Colonials in South Africa, Édimbourg, Blackwood, 1907. Parmi les pertes, on dénombre 89 Canadiens tués dans les régiments du Canada, 252 blessés et 135 morts dans des accidents ou en raison de maladies. Par ailleurs, 22000 Britanniques, 25000 Boers et 12000 autochtones perdent la vie : Packenham, The Boer War, p. xv.

${ }^{84}$ T.G. Marquis, "The South African War", dans Canada in the Great World War, vol. 1, Toronto, Morang, 1917, p. 285 à 289 ; Evans, The Canadian Contingents and Canadian Imperialism, p. 1, 14, 64, 245. Morrison, With the Guns in South Africa,p. 12 ; E.B. Biggar, The Boer War: Its Causes, and its interests to Canadians, Toronto, Biggar, 1899, p. 29-31. Labat, dans Le livre d'or, propose une interprétation favorable de l'appui des francophones à l'empire. L'exception la plus remarquable est Goldwin Smith, qui s'exprime dans In the Court of History: An Apology for Canadians who were opposed to the South African War, Toronto, Tyrell, 1902, p. 51.

${ }^{85}$ Bibliothèque et Archives Canada, documents de Denison, vol. 9 et 10, Denision à Chamberlain, 16 février 1901. Selon Bourassa, «L'impérialisme anglais est un régime d'accaparement et de domination militaire, né de l'expansion exagérée de la puissance anglaise et nourri de cet orgueil stupide, 
brutal et vantard qu'on nomme jingoisme. » Voir Henri Bourassa, « GrandeBretagne et Canada : questions actuelles ; conférence au Théâtre national français, Montréal, le 20 oct. 1901 », Montréal, Pionnier, [1902], p. 3.

${ }^{86}$ Brereton Greenhous dit que, durant la guerre, les Canadiens se sont acquis la réputation d'être des " combattants rudes et fiables aux yeux des Britanniques et des Boers ». Brereton Greenhous, "The South African War », dans J. Marteinson, dir., We Stand on Guard: An Illustrated History of the Canadian Army », Montréal, Ovale, 1992, p. 80.

${ }^{87}$ Stephen J. Harris, Canadian Brass: the Making of a Professional Army, 1860 1939, Toronto, University of Toronto Press, 1980, 271 p. Le Canadian Patriotic Fund, créé le 12 janvier 1900 pour aider les soldats handicapés, ne compte qu'un seul francophone dans son comité exécutif : le juge Désiré Girouard, qui rejoint Laurier (vice-président) et 31 anglophones. Bibliothèque et Archives Canada, RG 7 G21, vol. 129, dossier 233, dossiers numérotés du gouverneur général

${ }^{88}$ Il n'y a pas de forte tradition militaire au Québec depuis plus d'un siècle. Avant la guerre, comme le font remarquer Jean Pariseau et Serge Bernier, les zouaves pontificaux ont néanmoins fait la preuve que les Canadiens français " étaient tout aussi enthousiastes envers le service militaire que leurs compatriotes anglophones lorsqu'il s'agissait d'une cause qui leur était chère ». Pariseau et Bernier, Le Bilinguisme, p. 67. Voir aussi Roch Legault, Une élite en déroute : Les militaires canadiens après la Conquête, Montréal, Athéna, 2002, 202 p. ${ }^{89}$ Les francophones ont également participé à la campagne du Nord-Ouest, mais les critiques de la presse canadienne anglaise à l'égard de leur performance dans l'Ouest et, plus tard, en Afrique du Sud refroidissent beaucoup leur enthousiasme pour la milice canadienne. Voir Morton, « Le Canada français et la milice canadienne »; Pierre Vennat et Michel Litalien, Carabiniers et voltigeurs contre Louis Riel: histoire militaire et politique méconnue, Montréal, Méridien, 2003, 308 p.

${ }^{90}$ Bibliothèque et Archives Canada, documents de Minto, vol. 17, Hutton à Minto, 18 et 28 juin 1899, 10 février 1902. Voir aussi Desmond Morton, Ministers and Generals: Politics and the Canadian Militia, 1868-1904, [Toronto], University of Toronto Press, p. 158 ; Pariseau et Bernier, Le Bilinguisme, p. 64 à 66 .

${ }^{91}$ Direction de l'histoire et du patrimoine, Cummins, Lessard, p. 129. Musée canadien de la guerre, documents d'Otter, vol. 101, Hughes à Otter, 29 septembre 1899. Bibliothèque et Archives Canada, documents de Laurier, C-769- 
71, 4 octobre 1899 au 27 décembre 1899 : Hughes propose d'augmenter le nombre de troupes (mai-juillet 1899) et sa concurrence avec Hutton. Miller, Painting the Map Red, p. 205. Concernant Lessard et Hughes, voir Ronald G. Haycock, Sam Hughes: The Public Career of a Controversial Canadian, 1885-1916, Waterloo, Wilfrid Laurier University Press en collaboration avec le Musée canadien de la guerre, le Musée canadien des civilisations et les Musées nationaux du Canada, 1986, p. 22 à 27, 140, 157 à 159, 189, 213 à 218.

${ }^{92}$ La Presse, 21 novembre 1899, p. 1.

${ }^{93}$ J.M. Hitsman et J.L. Granatstein, Broken Promises: A History of Conscription in Canada, Toronto, Oxford University Press, 1977, 281 p. ; Elizabeth Armstrong, Le Québec et la Crise de la Conscription 1917-1918, Montréal, VLB, 1998, $293 \mathrm{p}$.

${ }^{94}$ Charles Taylor, Reconciling the Solitudes: Essays on Canadian Federalism and Nationalism, Montréal et Kingston, McGill-Queen's University Press, 1993, p. 195-196 ; Fernand Dumont, Genèse de la Société québécoise, Montréal, Boréal, 1993, 393 p. ; Ronald Rudin, Making History in Twentieth-Century Quebec, Toronto, University of Toronto Press, 1997, p. 191 ; Jocelyn Létourneau, "Québec d'après guerre et mémoire collective de la technocratie ", Cabiers internationaux de sociologie, vol. 90 (1991), p. 67-87.

${ }^{95}$ Bourassa croit que la montée de la Ligue nationaliste est directement liée à la participation du Canada à la guerre en Afrique du Sud : Le Devoir, 14 mai 1913, cité dans Miller, Painting the Map Red, p. 444.

${ }^{96}$ Laurier, inspiré par son désir de réduire les tensions linguistiques provoquées par la guerre, continue de jauger l'enthousiasme des Canadiens anglais et la tolérance des Canadiens français. Bibliothèque et Archives Canada, documents de Bourassa, M-722, 20 août 1900. Lorsque Bourrassa prévient Laurier que les deux extrêmes doivent être évités (ignorer l'opinion publique et la suivre aveuglément), Laurier répond qu'il ne voit pas d'alternative et que la solution de Bourassa serait catastrophique. Voir aussi la correspondance des 14 et 20 août 1900, dans Corcoran, « Bourassa », p. 427. Laurier ajoute, devant le Parlement, que le gouvernement ne devrait pas suivre l'opinion publique si celle-ci n'est pas honorable, mais que, si elle est juste et honorable, pourquoi pas? (Chambre des Communes, Débats, 13 mars 1900, p. 1833). Pour Laurier c'est une question de tactique. Il demande à Bourassa : "Quelle attitude, dites-moi, les Canadiens français doivent-ils prendre dans la Confédération? Il faut ou qu'ils s'isolent, fassent bande à part, ou qu'ils marchent à la tête de la Confédération. Il faut qu'ils choisissent entre l'impérialisme anglais, ou l'impérialisme américain. Je ne vois pas d'autre alternative. » Bibliothèque et 
Archives Canada, documents de Bourassa, M-722, 38692, Laurier à Bourassa, 2 novembre 1899. Pour Bourassa, c'est une question de principe, et les dirigeants francophones doivent diriger leur peuple sans compromis. Bibliothèque et Archives Canada, documents de Laurier, C-770, 38692, Bourassa à Laurier, 4 novembre 1899.

${ }^{97}$ Rudin, Making History in Twentieth-Century Quebec, p. 220-221.

${ }^{98}$ Taylor, Reconciling the Solitudes.

${ }^{99}$ Miller, Painting the Map Red, p. 16 à 19, 26 à 30, 441. Il fait remarquer (p. xii) que les historiens du $\mathrm{XX}^{\mathrm{c}}$ siècle ne se sont guère intéressés à cette guerre. Voir aussi Barker, «Laurier, French Canada and the Boer War», p. 66, 72 ; Greenhous, "The South African War », p. 56, 64 ; Brown et Cook, Canada, 1896-1921: a Nation Transformed, p. 39 ; Reid, Our Little Army in the Field, p. 13, 21. Même les arguments de Bourassa semblent de plus en plus pro-canadiens et non antibritanniques. Voir Corcoran, "Henri Bourassa et la guerre sud-africaine", p. 440 ; Greenhous, Dragoon, p. 74 ;.Page, "The Boer War and Canadian Imperialism », p. 13. Barker, «Laurier », p. iv.

${ }^{100}$ Carman Miller, "Canada and the Boer War ", série Histoire du Canada en images, vol. 24, Musée national de l'homme, Ottawa, 1980, p. 3 ; Pariseau et Bernier, Les Canadiens français et le bilinguisme dans les Forces armées canadiennes, $\mathrm{p}$. 12, 66 ; Pelletier, « Les Canadiens français et la Guerre des Boers », p. 49 à 66, 98.

${ }^{101}$ Linteau et al., Québec, p. 598-599 ; Lionel Groulx, Histoire du Canada Français, tome 2, Montréal, Fides, 1970, p. 328 ; Michel Brunet, Québec-Canada anglais, Montréal, HMH, 1969, p. 167-168. 